\title{
Pharmacological interventions for drug-using offenders: an update to a systematic review and meta-analysis
}

\author{
J. M. Glanville ${ }^{1}$ - A. E. Perry ${ }^{2}$ D $\cdot$ M. Martyn-St James ${ }^{3} \cdot$ C. Hewitt ${ }^{2} \cdot$ S. Swami $^{6}$. $^{-}$ \\ K. Wright ${ }^{5}$ - L. Burns ${ }^{2}$ - C. Pearson ${ }^{2}$ - A. Aboaja ${ }^{4}$ - P. Thakkar ${ }^{4}$ - K. M. S. Kumar ${ }^{4}$. \\ M. Bunney ${ }^{1}$
}

Accepted: 7 December 2020/ Published online: 15 February 2021

(C) The Author(s) 2021

\begin{abstract}
This updated systematic review assesses the effects of pharmacological interventions for drug-using offenders.

Methods Systematic review protocols and conventions of the Cochrane Collaboration were followed to identify eligible studies. Studies were pooled in a meta-analysis to assess the impact of pharmacological interventions on drug use and criminal activity. An economic appraisal was conducted.

Results The search strategies identified 22 studies containing 4372 participants. Metaanalyses revealed a small statistically significant mean difference favouring pharmacological interventions relative to psychological interventions in reducing drug use and criminal activity. When comparing the drugs to one another there were no significant differences between those included (methadone versus buprenorphine, naltrexone and cyclazocine).
\end{abstract}

Conclusion Overall, the findings of this review suggest that methadone and naltrexone may have some impact on reducing drug use and reincarceration. Individual pharmacological drugs had differing (generally non-significant) effects. One study identified serious adverse events. Three studies reported cost and consequences information sufficient to conduct a full economic analysis but this was not comprehensive enough to be able to make judgements across all treatment options. Full economic analyses should be encouraged. The study findings were limited mainly to male adult offenders.

Keywords Systematic review · Drug treatment · Economic appraisal · Offenders · Crime
A. E. Perry
amanda.perry@york.ac.uk

Extended author information available on the last page of the article 


\section{Introduction}

Offenders as a socially excluded group of people demonstrate significant drug use and subsequent health problems. Studies investigating the prevalence of drug dependence in UK prisons report variable figures of 10\% (Gunn et al., 1991), 39\% (Brooke et al., 1996) and 33\% (Mason et al., 1997). In a review article, the estimated prevalence of drug abuse among male and female prisoners was estimated to be 10 to $48 \%$ and 30 to $60 \%$, respectively (Fazel et al., 2006). A recent survey carried out on the Swedish criminal justice system found that $70 \%$ of all individuals had substance abuse problems (including alcohol, drugs or both), with a slightly lower percentage among females compared with the whole group (67\%) (Klientkartlaggningen, 2015). These numbers can be compared with the general population in Sweden where $1 \%$ of all men and $0.5 \%$ of all women fulfil the criteria for drug dependence or abuse (Ramstedt et al., 2013). Similar trends have been reported elsewhere. In France, $30 \%$ of prison inmates are heroin addicted, and in Australia, 59\% of prison inmates report injecting (primarily heroin) drug use histories. In 2018 in the USA, an estimated 6,410,000 persons were held in prisons or jails, or were on probation or parole (Maruschak and Minton, 2020). Of the prison population, $85 \%$ are likely to have an active substance use disorder or were incarcerated for a crime involving drugs or drug use according to the US National Institute on Drug Abuse (National Institute on Drug Abuse (NIDA), 2020). In the US, it is recognised that many offenders need treatment to tackle their drug use (Lo and Stephens, 2000). The link between drug use, subsequent health, social and criminological consequences is well documented in the literature (Michel and Maguet, 2005), and offenders have a high risk of death from opioid overdose within 2 weeks of release from prison (Binswanger et al., 2007, Bird and Hutchinson, 2003, Kinner et al., 2013, Merrall et al., 2010). Substance use disorders are linked to criminal behaviour and are a significant burden on the criminal justice system. Approximately $30 \%$ of acquisitive crime is committed by individuals supporting drug use with the use of criminal acts (Magura et al., 1995).

Internationally, methadone maintenance has been the primary choice for chronic opioid dependence in prisons and jails, including those in the Netherlands, Australia, Spain and Canada. As a result, it is increasingly implemented in the criminal justice setting (Moller et al., 2007, Stallwitz and Stover, 2007) with the exception of the US which has not generally endorsed the use of methadone treatment. For example, of the 30,000 individuals a year who enter prison or jail nearly $90 \%$ of those receiving prescribed methadone are forced to stop or taper off this treatment (Fiscella et al., 2004. Reasons for this lack of expansion suggest that public opinion and that of the criminal justice system providers consider methadone treatment as substituting one addiction for another. In contrast, buprenorphine appears not to carry the same social stigma associated with methadone treatment and has been used in France, Austria and Puerto Rico (Catania, 2003, Garcia et al., 2007, Gordon et al., 2017, Reynaud-Maurupt et al., 2005). This is noted because unlike methadone it can be administered on alternate days, a feature that would make buprenorphine use more efficient in correctional settings than methadone (Garcia et al., 2007). Naltrexone treatment has shown some promising findings, but associated problems surrounding high attrition and low medication compliance in the community and high mortality rates pose concerns (Gibson and Degenhardt, 2007, Minozzi et al., 2011). In 2005 naloxone was added to the UK's 
exempt list of prescription-only medicines that could be administered to save life in an emergency to reverse heroin/opioid overdose (Strang et al., 2006). Use of extendedrelease naltrexone may also be appealing and beneficial to people who are unlikely to access opioid-agonist maintenance treatment or who prefer a relapse-prevention treatment (Lee et al., 2016). Trials conducted in the criminal justice setting are still lacking, and continuity of care is considered crucial in the treatment of drug-using offenders who transition between prison and the community.

A growing body of evidence shows the effects of pharmacological interventions for drug use among the general population (see Table 1 for a summary of the evidence). Existing reviews have focused on naltrexone maintenance treatment for opioid dependence (Amato et al., 2005, Lobmaier et al., 2008, Minozzi et al., 2011), the efficacy of methadone (Faggiano et al., 2003, Marsch, 1998, Mattick et al., 2009) and buprenorphine maintenance (Mattick et al., 2009). Recent guidance has been provided from the National Institute for Health and Clinical Excellence (NICE) on evidencebased use of naltrexone, methadone and buprenorphine for the management of opioid dependence (National Institute for Health and Care Excellence (NICE), 2007a, National Institute for Health and Care Excellence (NICE), 2007b, National Institute for Health and Care Excellence (NICE), 2019). Five Cochrane reviews (including 52 studies) reported on the effectiveness of opiate methadone therapies (Amato et al., 2005). Findings showed that methadone maintenance therapies at appropriate doses were most effective in retaining participants in treatment and in suppressing heroin use, but evidence of effectiveness for other relevant outcome measures such as criminal activity was weak and was not systematically evaluated. Naloxone use is also found to work in a similar manner by blocking the euphoria (reinforcement) sought by the user and thereby reducing the drug-seeking behaviour and diminishing the risk of physical dependence of heroin use (Kurland et al., 1975). Extended-release naltrexone uses the same mechanism as naloxone but gradually releases a sufficient amount to block the euphoria up to 1 month after an injection (Lee et al., 2016).

Systematic reviews evaluating treatment programmes for offender populations have focused on evaluating treatments in one setting such as community-based programmes (Mitchell et al., 2012a, Mitchell et al., 2012b), or have based their evidence on literature from one country (Chanhatasilpa et al., 2000, Egg et al., 2000), or a number of specific treatments (Mitchell et al., 2006). Pharmacological systematic reviews of offender treatment appear to be sparse. We identified two previous reviews: one focusing on specific drug- and property-related criminal behaviours in methadone maintenance treatment (Marsch, 1998), and one an evaluation of the effectiveness of opioid maintenance treatment (OMT) in prison and post release (Hedrich et al., 2012). The latter of these two reviews identified six experimental studies up until January 2011 (Hedrich et al., 2012). The authors found that OMT in prison was significantly associated with reduced heroin use, injecting and syringe sharing. The use of pre-release OMT was also found to have important implications for associated treatment uptake after release, but the impact on criminal activity was equivocal. Similar findings have been shown with the use of extended-release naltrexone (XR-NTX) which showed that use of XR-NTX before release might reduce opioid overdose, increase abstinence and antagonist medication coverage during the high-risk initial weeks after release in comparison with referral for XR-NTX treatment in the community after release (Friedmann et al., 2018).

Many people under the care of the criminal justice system have a drug misuse 


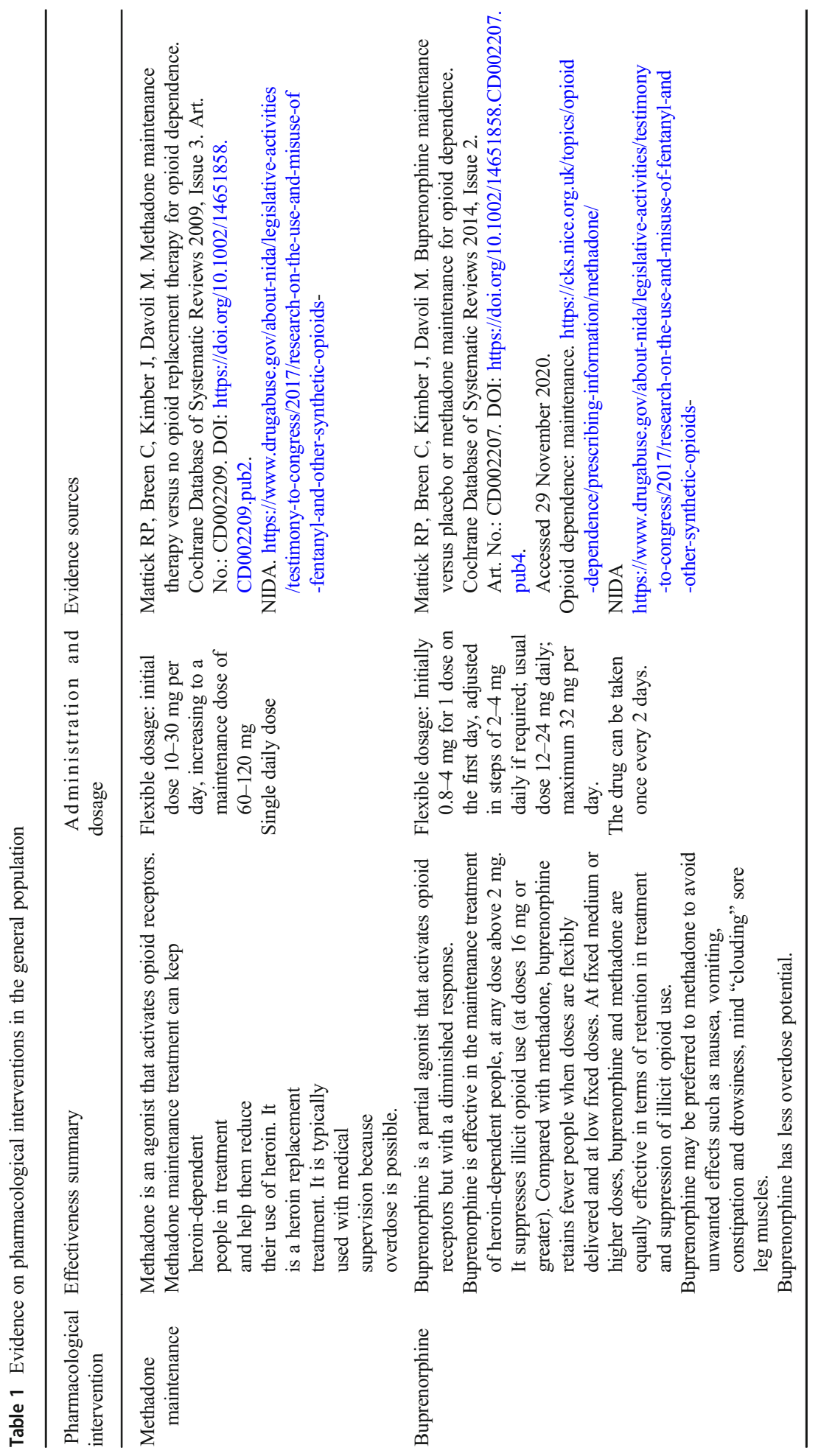




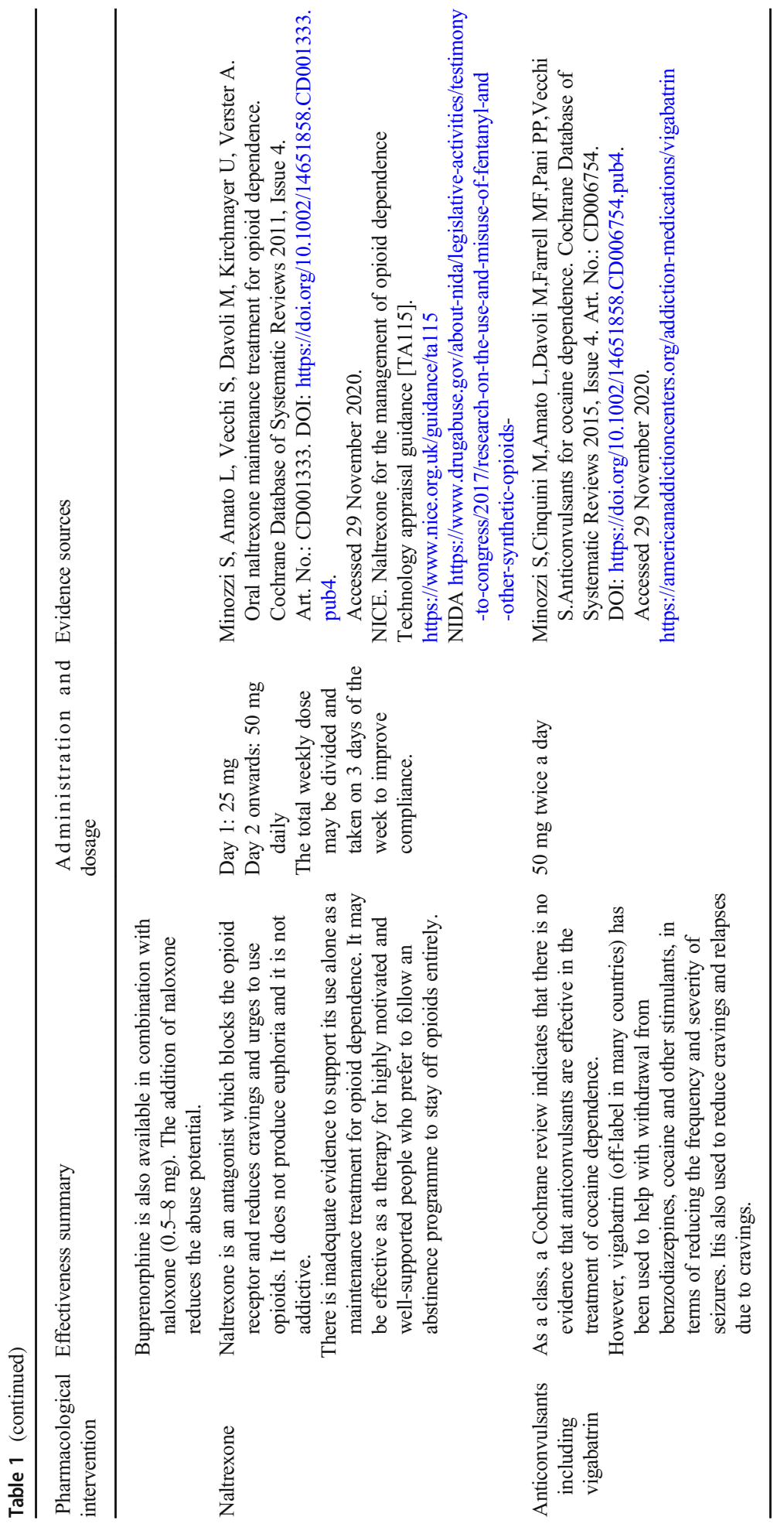


problem (Fazel et al., 2015). Whilst previous research has evaluated treatment programmes for offenders more broadly, we know little about the challenges, treatment and rehabilitation opportunities for offenders with pharmacological interventions aimed at reducing drug use and criminal activity. This includes consideration of when and where to offer treatment along the criminal justice pathway to maximise scarce resources and maximise the effectiveness of treatment. We therefore believe that a systematic review evaluating existing evidence from randomised controlled trials (RCTs) might be helpful in identifying treatments for reducing drug use and criminal activity in this vulnerable population. In principle, the RCT design eliminates the threat to internal validity providing there is a sufficiently large number of units assigned as the experimental and control conditions and methods to reduce bias are implemented (Weisburd, 2010).

Given the importance of relating economic cost to a reduction in drug use and related offending behaviour, good-quality economic evidence will help inform strategies which represent the best use of limited resource (Higgins and Green, 2011). In this review, we use the Drummond checklist (Drummond et al., 2005) to evaluate and document the availability of resource information within the studies. The Drummond checklist is a widely used tool to assess the quality of economic evaluation studies. It assesses whether the study has a well-defined question, describes the competing programmes, provides information on the effectiveness of the programmes, presents all of the important and relevant costs and consequences for each programmes and assesses whether costs and consequences of the programmes were accurately measured and assigned credible values (and discounted appropriately). The checklist also assesses any incremental cost-effectiveness analysis and sensitivity analysis around the costs and consequences.

This systematic review has five primary research questions: (1) Do pharmacological treatments for drug-using offenders reduce drug use? (2) Do pharmacological treatments for drug-using offenders reduce criminal activity? (3) Does the treatment setting (e.g. court, community, prison/secure establishment) affect outcome(s) of pharmacological treatments? (4) Does one type of pharmacological treatment perform better than one other? (5) What are the comparative costs and cost-effectiveness of the available interventions?

The systematic review was guided by a protocol which followed Cochrane approaches (Higgins and Green, 2011) to producing systematic reviews and an earlier version has been published as a Cochrane review (Perry et al., 2015).

\section{Methods}

Studies included in the review had to meet a number of different criteria including the study design, the type of participants and type of interventions. Studies had to report pre-specified outcome measures to avoid the possibility of any subsequent bias in choosing outcomes. The eligibility criteria are summarised below and described in detail in Table 2.

Studies of illicit drug-using offenders were included in the review regardless of the gender, age, psychiatric history or ethnicity of the study participants. Eligible interventions were those designed, wholly or in part, to eliminate or prevent relapse to drug use 
Table 2 Summary of eligibility criteria

\begin{tabular}{|c|c|c|}
\hline & Inclusion criteria & Exclusion criteria \\
\hline Population & $\begin{array}{l}\text { - Illicit drug-using offenders were included in } \\
\text { the review regardless of gender, age, psy- } \\
\text { chiatric history, or ethnicity. } \\
\text { - Offenders were defined as individuals who } \\
\text { were involved in the Criminal Justice } \\
\text { System (CJS). } \\
\text { - Individuals could reside in special hospitals, } \\
\text { prisons, the community or who were } \\
\text { diverted from court or placed on arrest } \\
\text { referral schemes for treatment. } \\
\text { - The study setting could change throughout } \\
\text { the process of the study. For example, } \\
\text { people involved in the criminal justice } \\
\text { system could begin in prison but progress } \\
\text { through a work release project into a } \\
\text { community setting. } \\
\text { - Illicit drug use referred to individuals using } \\
\text { drugs occasionally or those who were } \\
\text { considered drug dependent. }\end{array}$ & $\begin{array}{l}\text { Studies where part of the population were not } \\
\text { involved in the CJS. } \\
\text { Studies where the population were } \\
\text { alcohol-using as opposed to drug-using of- } \\
\text { fenders. }\end{array}$ \\
\hline Intervention & $\begin{array}{l}\text { - Eligible interventions were those designed, } \\
\text { wholly or in part, to eliminate or prevent } \\
\text { relapse to drug use or criminal activity, or } \\
\text { both, among participants. We defined } \\
\text { relapse as individuals who may have } \\
\text { returned to an incarcerated setting, or had } \\
\text { subsequently been arrested or had relapsed } \\
\text { back into drug misuse, or both. We included } \\
\text { a range of different types of interventions in } \\
\text { the review. } \\
\text { - Experimental interventions included in the } \\
\text { review were any pharmacological } \\
\text { intervention (e.g. buprenorphine, } \\
\text { methadone). } \\
\text { - Control interventions eligible for the review } \\
\text { were no treatment or waiting list controls or } \\
\text { minimal and/or alternative treatment or } \\
\text { treatment as usual: } \\
\text { Minimal or alternative treatment might include } \\
\text { reporting use of a similar intervention but at } \\
\text { a less intense level, or using a different } \\
\text { theoretical approach but with the same } \\
\text { components and/or a different alternative } \\
\text { intervention. } \\
\text { Treatment as usual included any study that } \\
\text { reported a combination and/or component } \\
\text { of a (i) psychological based intervention } \\
\text { (e.g. anger management, motivational } \\
\text { interviewing, counselling, aggression } \\
\text { replacement, family therapy), (ii) an educa- } \\
\text { tional programme (e.g., health, substance } \\
\text { abuse education on risky behaviour) and or } \\
\text { (iii) life skills (e.g., financial planning, em- } \\
\text { ployment skills, computer skills and inter- } \\
\text { personal skills in interviews. }\end{array}$ & $\begin{array}{l}\text { Studies of alcohol misuse only were not } \\
\text { eligible. } \\
\text { Studies of interventions with drug-using of- } \\
\text { fenders that were not focused on reducing } \\
\text { drug use. }\end{array}$ \\
\hline
\end{tabular}


Table 2 (continued)

\begin{tabular}{|c|c|c|}
\hline & Inclusion criteria & Exclusion criteria \\
\hline $\begin{array}{l}\text { Outcomes } \\
\text { measures }\end{array}$ & $\begin{array}{l}\text { - Primary outcomes were drug use measures } \\
\text { (self-reported drug use and biological drug } \\
\text { use), self-reported or official report of } \\
\text { criminal activity and adverse effects. } \\
\text { - Secondary outcomes were resource use, costs } \\
\text { and cost-effectiveness related to the } \\
\text { interventions, health care activity, criminal } \\
\text { activity and productivity. }\end{array}$ & \\
\hline $\begin{array}{l}\text { Study } \\
\text { design }\end{array}$ & - RCTs were the eligible study design & $\begin{array}{l}\text { Studies that did not report data in a format that } \\
\text { allowed us to generate post } \\
\text { dichotomous/continuous outcomes }\end{array}$ \\
\hline Limits & $\begin{array}{l}\text { - No date or language limits will be applied to } \\
\text { the searches }\end{array}$ & \\
\hline
\end{tabular}

or criminal activity, or both, among participants. We defined relapse as individuals who may have returned to an incarcerated setting, or had subsequently been arrested or had relapsed back into drug misuse, or both. The primary outcomes investigated in the review were drug use (self-reported drug use measures and biological drug use measures), self-reported or official report of criminal activity (dichotomous and continuous data for arrests, reincarceration and parole violations) and adverse effects. Secondary outcomes were resource use, costs and cost-effectiveness related to the interventions, health care activity, criminal activity and productivity. Randomised controlled trials (RCTs) were the eligible study design and no date or language limits were applied to this review.

\section{Search strategy for identification studies}

The original searches for this review are reported in Perry (Perry et al., 2006, Perry et al., 2015) and were subsequently updated in January 2018 to cover the period 2014 to 2018 and then updated further again in February 2019 to cover the period 2018 to 2019. We searched 12 databases: ASSIA, CINAHL, Cochrane Central Register of Controlled Trials (CENTRAL), Criminal Justice Abstracts, Embase, Health Management Information Consortium (HMIC), LILACS, MEDLINE, PAIS, PsycINFO, Science Citation Index and Social Science Citation Index. The full search strategies for each database are included in the Appendix.

To identify economic studies the topic specific searches of nine databases (ASSIA, CENTRAL, CINAHL, Criminal Justice Abstracts, HMIC, LILACS, PAIS, Science Citation Index, Social Science Citation Index) would have identified all potential RCTs and economic evaluations included in the review. For the Embase, MEDLINE and PsycINFO databases the search strategy incorporated an RCT filter to manage the number of identified and relevant citations. This approach may have excluded potential economic evaluations identified from these three databases and is therefore a limitation of the review.

We developed individual search strategies for each database and made use of any controlled vocabulary available for each database. We combined the subject search 
terms with methodological search filters designed to identify RCTs, where available. Additional search approaches were employed including checking the reference lists of all retrieved articles for further references, searching catalogues of relevant organisations and contacting experts to obtain recommendations of other published or unpublished studies relevant to the review. All references were managed in EndNote (Clarivate, 2020) and subsequently Covidence (Veritas Health Innovation, 2013) software.

\section{Screening and coding process}

A team of reviewers independently inspected the titles and abstracts for potential inclusion in the review. Each record was assessed by two reviewers. The full articles were obtained for each record which seemed likely to be eligible and the full text was assessed for eligibility by two reviewers independently. In the case of disagreement, a third independent reviewer helped to arbitrate. The screening criteria are provided in the Appendix.

We used data extraction forms to standardise the reporting of data from all studies obtained as potentially relevant. Two reviewers independently extracted data and subsequently checked them for agreement. The narrative tables included a presentation of the study details (e.g. author, year of publication and country of study origin), study methods (e.g. random assignment), participants (e.g. number in sample, age, gender and ethnicity), interventions (e.g. description, duration, intensity and setting), outcomes (e.g. description, follow-up period and reporting mechanism) and notes (e.g. country and funding).

\section{Measures of treatment effect}

Studies were combined as mean differences (MD) with $95 \%$ confidence intervals in the meta-analyses for continuous outcomes measured on the same scale and standardised mean difference (SMD) for outcomes measured on different scales. Higher scores for continuous measures are representative of greater harm. We present dichotomous outcomes as risk ratios (RR), with 95\% confidence intervals (CIs).

\section{Statistical issues}

To avoid double counting of outcome measures (e.g. arrest and parole violation) and follow-up time periods (e.g. 12, 18 months), we checked all trials to ensure that multiple studies reporting the same evaluation did not contribute towards multiple estimates of programme effectiveness. We followed Cochrane guidance and we combined intervention and control groups to create a single pair-wise comparison. Where this was not appropriate we selected one treatment arm and excluded the others. We attempted to contact the study authors via email where missing data occurred in the original publication.

We assessed heterogeneity using the $\mathrm{I}^{2}$ statistic and $\mathrm{Chi}^{2}$ statistic (Higgins and Green, 2011). We regarded heterogeneity as substantial if the $\mathrm{I}^{2}$ was greater than $50 \%$ or the $p$ value was lower than 0.10 for the $\mathrm{Chi}^{2}$ test for heterogeneity (Deeks et al., 2017). Following specific Cochrane guidance (Deeks et al., 2017), we 
distinguished the following values to denote no important, moderate, substantial and considerable heterogeneity, respectively: 0 to $40 \%, 30$ to $60 \%, 50$ to $90 \%$ and 75 to $100 \%$.

\section{Risk of bias assessment}

The review team assessed risk of bias in all included studies using risk of bias assessment criteria recommended in the Cochrane Handbook (Higgins and Green, 2011). Further detail on risk of bias assessment is provided in the Appendix.

\section{Data synthesis}

The Revman software package (The Nordic Cochrane Centre (The Cochrane Collaboration), 2014) was used to perform a series of meta-analyses for continuous and dichotomous outcome measures. A random effects model was used to account for participants coming from different underlying populations. We used the transformations as laid down by the Cochrane Handbook (Higgins and Green, 2011) for continuous outcomes. For conversions of standard error into standard deviations and the calculation of standard deviations calculated from $95 \%$ confidence intervals (CIs) we used the standard equations set out in Fig. 1.

\section{Economic appraisal}

Economic or resource use information was assessed using the Drummond classification scheme (see Appendix) (Drummond et al., 2005). This criterion was applied by an economist to indicate the costs and the consequences of the intervention(s) relevant to various public sectors. These included healthcare, criminality, labour force participation or other public goods. According to Drummond, studies containing information on the economics of the intervention can be classified as full economic evaluation studies or partial economic evaluation studies (Drummond et al., 2005). A full economic evaluation is a comparative analysis of two or more interventions in terms of both resource

\begin{tabular}{|lccc|}
\hline & $\begin{array}{c}\text { Group 1 } \\
\text { (e.g. males) }\end{array}$ & $\begin{array}{c}\text { Group 2 (e.g. } \\
\text { females) }\end{array}$ & Combined groups \\
Sample & $\mathrm{N}_{1}$ & $\mathrm{~N}_{2}$ & $\mathrm{~N}_{1}+\mathrm{N}_{2}$ \\
size & $\mathrm{M}_{1}$ & $\mathrm{M}_{2}$ & $\frac{\mathrm{N}_{1} \mathrm{M}_{1}+\mathrm{N}_{2} \mathrm{M}_{2}}{\mathrm{~N}_{1}+\mathrm{N}_{2}}$ \\
Mean & $\mathrm{SD}_{1}$ & $\mathrm{SD}_{2}$ & $\sqrt{\frac{\left(N_{1}-1\right) \mathrm{SD}_{1}{ }^{2}+\left(\mathrm{N}_{2}-1\right) \mathrm{SD}_{2}{ }^{2}+\frac{N_{1} N_{2}}{N_{1}+N_{2}}\left(\mathrm{M}_{1}{ }^{2}+\mathrm{M}_{2}{ }^{2}-2 \mathrm{M}_{1} \mathrm{M}_{2}\right)}{\mathrm{N}_{1}+\mathrm{N}_{2}-1}}$ \\
$\mathrm{SD}$ & & &
\end{tabular}

$$
\begin{aligned}
& S D=S E \times \sqrt{N} \\
& S D=\sqrt{N} \times(\text { upper limit }- \text { lower limit }) / 3.92
\end{aligned}
$$

Fig. 1 Statistical treatment 
use (or costs) and outcomes (or consequences) (Drummond et al., 2005). Full economic evaluations can be further classified as cost-benefit analysis, cost-effectiveness analysis and cost-utility analysis based on consequences measured in monetary units, physical units (e.g. mortality) and utilities (e.g. quality-adjusted life years (QALYs)), respectively. Evaluations that are not comparative or do not consider both costs and consequences are classified as partial evaluations (e.g. cost-outcome description or cost analysis). Studies containing economic information using the Drummond criteria were identified, and information related to resource use and perspectives were recorded.

\section{Search results}

Searches for this review were first conducted in Perry (Perry et al., 2006, Perry et al., 2015) and were last updated in February 2019. The searches identified a total of 16,786 records and 988 additional records were identified from other sources. Following deduplication, 9657 records were screened using information in the title and abstract. Of these, 9406 records were excluded. We acquired the remaining 251 full papers for assessment and excluded 237 of these. A total of 22 studies were finally deemed eligible for the review and were included in the qualitative synthesis, with 19 of them included in the meta-analyses. The 22 included studies randomised 4372 participants from research published between 1969 and 2019. Fig. 2 shows the PRISMA flow diagram of the numbers of records included and excluded at each stage of the selection process.

Fourteen of the 22 trials were conducted in the US and three in the UK. The other 5 studies were conducted in Iran, Australia, Norway, Sweden and Mexico. Twenty-one of the 22 studies included male adult drug-using offenders and 1 study recruited only female participants (Cropsey et al., 2011). In 2 studies, the percentage of each gender was not reported (Kurland et al., 1975, Wright et al., 2011).

The age of study participants ranged from 17 to 55 years. In 7/22 (32\%) studies, the majority of participants were of white ethnic origin. The studies were divided by setting into community $(n=9)$ (Brodie et al., 2009, Brown et al., 2013, Cornish et al., 1997, Coviello et al., 2010, Cropsey et al., 2011, Hanlon et al., 1975, Hanlon et al., 1977, Kurland et al., 1975, Lee et al., 2016), prison $(n=7)$ (Bayanzadeh, 2004, Dolan et al., 2003, Dole et al., 1969, Howells et al., 2002, Lobmaier et al., 2010, Rich et al., 2015, Wright et al., 2011) and those that started in a secure setting and continued into the community $(n=6)$ (Kinlock et al., 2005, Kinlock et al., 2007, Konstenius et al., 2014, Magura et al., 2009, McKenzie et al., 2012, Parmar et al., 2017).

Fifteen of the 22 trials evaluated outcomes at 6 months or less, 4 trials reported outcomes up to 12 months (Brown et al., 2013, Dole et al., 1969, Kinlock et al., 2007), 1 study reported outcomes at 18 months (Lee et al., 2015) and 1 reported outcomes at 4 years (Dolan et al., 2003).

Different outcome measures were presented for each study, and just over half of all studies reported 4 or more outcome measures. One study reported on criminal activity only (Cornish et al., 1997) and 8 trials reported on drug use only (Bayanzadeh, 2004, Brown et al., 2013, Cropsey et al., 2011, Dolan et al., 2003, Hanlon et al., 1977, Konstenius et al., 2014, Wright et al., 2011). Six studies did not report any information on adverse events, side effects from the drug or drug safety (Bayanzadeh, 2004, Dolan et al., 2003, Dole et al., 1969, Hanlon et al., 1975, Hanlon et al., 1977, Kurland et al., 


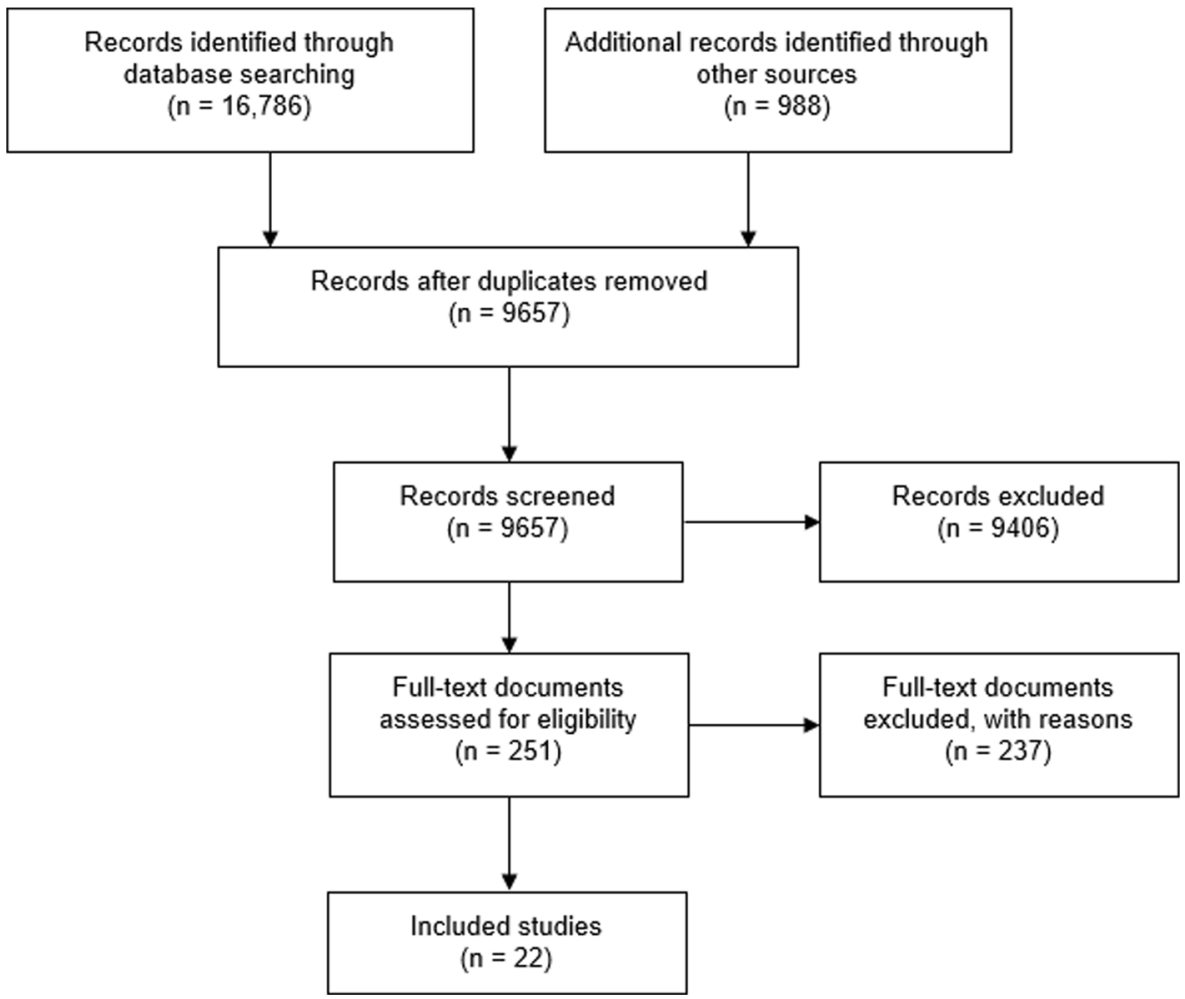

Fig. 2 PRISMA flow diagram

1975). Three studies referred to the measurement of adverse events but did not report them (Coviello et al., 2010, Cropsey et al., 2011, Wright et al., 2011). 4 studies reported resource and cost information (Magura et al., 2009, Murphy et al., 2017, Rich et al., 2015, Warren et al., 2006).

\section{Overview of studies}

\section{Meta-analyses}

Nineteen of the 22 studies provided data to potentially be included in 1 or more metaanalyses. The interventions report on agonistic pharmacological interventions (buprenorphine, methadone) and antagonistic pharmacological interventions (naltrexone and naloxone) compared with no intervention, other non-pharmacological treatments (e.g. counselling) and other pharmacological drugs (vigabatrin and cyclazocine). The comparisons are shown in Table 3 and in Fig. 3.

\section{Agonist (methadone) versus no intervention (placebo or waiting list control)}

Two studies (237 participants) (Cropsey et al., 2011, Dolan et al., 2003) showed no significant reduction in drug use using biological measures (RR 0.74, 95\% CI 0.53 to 
Table 3 Meta-analysis results by comparison and outcome

\begin{tabular}{llll}
\hline $\begin{array}{l}\text { Intervention comparison and } \\
\text { outcome(s) }\end{array}$ & $\begin{array}{l}\text { Studies (author/ } \\
\text { year) }\end{array}$ & $\begin{array}{l}\text { Number of } \\
\text { participants }\end{array}$ & $\begin{array}{l}\text { Effect size 95\% CI } \\
\text { (RR/SMD/ } \\
\text { MD) }\end{array}$
\end{tabular}

Agonist (methadone) vs no intervention (placebo or waiting list control)

\begin{tabular}{|c|c|c|c|c|c|}
\hline Drug use (objective) & $\begin{array}{l}\text { Cropsey et al. } 2011 \\
\text { Dolan et al. } 2003\end{array}$ & 237 & RR: 0.72 & $0.51,1.00$ & 0.05 \\
\hline $\begin{array}{l}\text { Self-reported drug use } \\
\text { (dichotomous) }\end{array}$ & $\begin{array}{l}\text { Cropsey et al. } 2011 \\
\text { Dolan et al. } 2003 \\
\text { Dole et al. } 1969\end{array}$ & 317 & RR: 0.61 & $0.31,1.18$ & 0.14 \\
\hline $\begin{array}{l}\text { Self-reported drug use } \\
\quad \text { (continuous) }\end{array}$ & $\begin{array}{l}\text { Dolan et al. } 2003 \\
\text { Kinlock et al. } 2005\end{array}$ & 459 & SMD: -0.64 & $-0.90,-0.39$ & $<0.0001$ \\
\hline $\begin{array}{l}\text { Criminal activity - arrests } \\
\text { (dichotomous) }\end{array}$ & Kinlock et al. 2005 & 62 & RR: 0.60 & $0.32,1.14$ & 0.12 \\
\hline $\begin{array}{l}\text { Criminal activity- } \\
\text { reincarnation } \\
\text { (dichotomous) }\end{array}$ & $\begin{array}{l}\text { Dolan et al. } 2003 \\
\text { Dole et al. } 1969 \\
\text { Kinlock et al. } 2005\end{array}$ & 472 & RR: 0.77 & $0.36,1.64$ & 0.50 \\
\hline $\begin{array}{l}\text { Criminal activity } \\
\text { (continuous) }\end{array}$ & Kinlock et al. 2005 & 51 & MD: -74.21 & $-133.53,14.89$ & 0.01 \\
\hline \multicolumn{6}{|c|}{ Agonist (methadone) vs partial agonist (buprenorphine) } \\
\hline $\begin{array}{l}\text { Self-reported drug use } \\
\text { (dichotomous) }\end{array}$ & $\begin{array}{l}\text { Magura et al. } 2009 \\
\text { Wright } 2001\end{array}$ & 370 & RR: 1.04 & $0.69,1.55$ & 0.87 \\
\hline $\begin{array}{l}\text { Self-reported drug use } \\
\text { (continuous) }\end{array}$ & Magura et al. 2009 & 81 & MD: 0.70 & $-5.33,6.73$ & 0.82 \\
\hline $\begin{array}{l}\text { Criminal activity- } \\
\text { reincarnation } \\
\text { (dichotomous) }\end{array}$ & Magura et al. 2009 & 116 & RR: 1.25 & $0.83,1.88$ & 0.28 \\
\hline \multicolumn{6}{|c|}{ Agonist (methadone) vs antagonist (naltrexone) } \\
\hline $\begin{array}{l}\text { Self-reported drug use } \\
\text { (continuous) }\end{array}$ & $\begin{array}{l}\text { Lobmaier et al. } \\
\quad 2010\end{array}$ & 44 & MD: 4.60 & $-3.54,12.74$ & 0.27 \\
\hline $\begin{array}{l}\text { Criminal activity- } \\
\text { reincarnation } \\
\text { (dichotomous) }\end{array}$ & $\begin{array}{l}\text { Lobmaier et al. } \\
\quad 2010\end{array}$ & 44 & RR: 1.10 & $0.37,3.26$ & 0.87 \\
\hline $\begin{array}{l}\text { Criminal activity } \\
\text { (continuous) }\end{array}$ & $\begin{array}{l}\text { Lobmaier et al. } \\
\qquad 2010\end{array}$ & 44 & $\mathrm{MD}:-0.50$ & $-8.04,7.04$ & 0.90 \\
\hline
\end{tabular}

Agonist (methadone) and non-pharmacological intervention vs non-pharmacological intervention

\begin{tabular}{|c|c|c|c|c|c|}
\hline $\begin{array}{l}\text { Self-reported drug use } \\
\text { (dichotomous) }\end{array}$ & $\begin{array}{l}\text { Bayanzadeh, } 2004 \\
\text { Kinlock et al. } 2007\end{array}$ & 203 & RR: 0.39 & $0.14,1.13$ & 0.08 \\
\hline \multicolumn{6}{|c|}{ Agonist (vigabatrin) vs placebo } \\
\hline $\begin{array}{l}\text { Number of negative urine } \\
\text { tests for cocaine }\end{array}$ & Brodie et al. 2009 & 103 & MD 2.10 & $-0.34,4.54$ & 0.09 \\
\hline $\begin{array}{l}\text { End of trial abstinence for } \\
\text { cocaine }\end{array}$ & Brodie et al. 2009 & 103 & RR: 1.06 & $0.28,4.01$ & 0.93 \\
\hline $\begin{array}{l}\text { End of trial abstinence for } \\
\text { marijuana }\end{array}$ & Brodie et al. 2009 & 103 & RR: 0.64 & $0.16,2.52$ & 0.52 \\
\hline
\end{tabular}

Antagonist (naloxone) vs no intervention (placebo or waiting list control)

$\begin{array}{llllll}\text { Self-reported drug use } & \text { Kurland et al. } 1975 & 333 & \text { RR: } 1.02 & 0.89,1.16 & 0.78 \\ & \text { Parmer 2016 } & & & \\ & \text { Hanlon et al. } 1977 & & & \end{array}$


Table 3 (continued)

\begin{tabular}{|c|c|c|c|c|c|}
\hline $\begin{array}{l}\text { Intervention comparison and } \\
\text { outcome(s) }\end{array}$ & $\begin{array}{l}\text { Studies (author/ } \\
\text { year) }\end{array}$ & $\begin{array}{l}\text { Number of } \\
\text { participants }\end{array}$ & $\begin{array}{l}\text { Effect size } \\
(\mathrm{RR} / \mathrm{SMD} / \\
\text { MD) }\end{array}$ & $95 \% \mathrm{CI}$ & $P$ value \\
\hline \multicolumn{6}{|c|}{ Antagonist (naltrexone) vs non-pharmacological intervention } \\
\hline $\begin{array}{l}\text { Criminal activity- } \\
\text { reincarnation } \\
\text { (dichotomous) }\end{array}$ & $\begin{array}{l}\text { Cornish et al. } 1997 \\
\text { Coviello et al. } 2010 \\
\text { Lee et al. } 2016\end{array}$ & 422 & RR: 0.55 & $0.31,0.99$ & 0.05 \\
\hline $\begin{array}{l}\text { Criminal activity_drugs } \\
\text { charges (dichotomous) }\end{array}$ & Coviello et al. 2010 & 63 & RR: 3.10 & $0.34,28.19$ & 0.32 \\
\hline $\begin{array}{l}\text { Drug use (objective)- } \\
\quad \text { opioids }\end{array}$ & $\begin{array}{l}\text { Coviello et al. } 2010 \\
\text { Lee et al. } 2016\end{array}$ & 371 & RR: 0.60 & $0.44,0.81$ & 0.0 \\
\hline $\begin{array}{l}\text { Drug use (objective)- } \\
\quad \text { cocaine }\end{array}$ & $\begin{array}{l}\text { Coviello et al. } 2010 \\
\text { Lee et al. } 2016\end{array}$ & 391 & RR: 1.47 & $0.67,3.23$ & 0.33 \\
\hline \multicolumn{6}{|c|}{ Mixed antagonist and agonist (cyclazocine) vs antagonist (naloxone) } \\
\hline Abstinent at 6 months & Hanlon et al. 1975 & 70 & RR: 1.14 & $0.46,2.81$ & 0.77 \\
\hline \multicolumn{6}{|c|}{ Agonist (methadone) vs forced tapered withdrawal from methadone } \\
\hline $\begin{array}{l}\text { Drug use and reincarceration } \\
1 \text { month post release }\end{array}$ & Rich et al. 2015 & 1078 & RR: 0.86 & $0.63,1.17$ & 0.23 \\
\hline $\begin{array}{l}\text { Any other drug use at } \\
1 \text { month post release }\end{array}$ & Rich et al. 2015 & 283 & RR: 1.05 & $0.83,1.34$ & 0.68 \\
\hline $\begin{array}{l}\text { Reincarceration at } 1 \text { month } \\
\text { post release }\end{array}$ & Rich et al. 2015 & 229 & RR: 0.92 & $0.39,2.16$ & 0.85 \\
\hline $\begin{array}{l}\text { Opioid use at } 1 \text { month post } \\
\text { release }\end{array}$ & Rich et al. 2015 & 283 & RR: 0.56 & $0.26,1.22$ & 0.14 \\
\hline Use of injectable illegal drug & Rich et al. 2015 & 283 & RR: 0.67 & $0.40,1.15$ & 0.15 \\
\hline
\end{tabular}

Agonist (methadone) prior to release with financial support vs agonist (methadone) on release with no financial support

Drug use (heroin) in past 30 days

McKenzie et al. $\quad 61$ 2012

Drug use (other opiates) in McKenzie et al. 61 past 30 days 2012 (dichotomous)

Drug use (crack/cocaine) in McKenzie et al. 61 past 30 days 2012 (dichotomous)

Drug use (marijuana) in past McKenzie et al 61 30 days (dichotomous) 2012

Drug use (polydrug) in past McKenzie et al. 61 30 days (dichotomous) 2012

Any drug injecting in the past 30 days

Criminal justice outcomes

Reincarceration (prison/jail/other)

McKenzie et al. 6 2012

McKenzie et al. $\quad 122$ 2012

McKenzie et al. 61 2012

Arrested in past 6 months

McKenzie et al. $\quad 61$ 2012

$\begin{array}{lll}\text { RR: } 0.53 & 0.22,1.25 & 0.14 \\ \text { RR: } 0.14 & 0.01,2.57 & 0.18\end{array}$

$61 \quad$ RR: $0.53 \quad 0.22,1.25 \quad 0.14$

$61 \quad$ RR: $0.48 \quad 0.10,2.45 \quad 0.38$

$61 \quad$ RR: $0.81 \quad 0.41,1.58 \quad 0.53$

$61 \quad$ RR: $0.36 \quad 0.11,1.24 \quad 0.11$

22 RR: $1.04 \quad 0.38,2.81 \quad 0.94$

$1 \quad$ RR: $0.48 \quad 0.10,2.45 \quad 0.38$

$1 \quad$ RR: $1.45 \quad 0.59,3.58 \quad 0.42$

$C I$ confidence interval, $R R$ risk ratio, $S M D$ standardised mean difference, $M D$ mean difference 
1.02) and three studies (317 participants) showed no significant reduction in selfreported drug use (RR 0.61, 95\% CI 0.31 to 1.18) (Cropsey et al., 2011, Dolan et al., 2003, Dole et al., 1969). Two studies (304 participants) showed a significant reduction in self-reported drug use (SMD - 0.57, 95\% CI - 0.80 to - 0.34) (Dolan et al., 2003, Kinlock et al., 2005). For criminal activity, one study (62 participants) showed no significant reduction in re-arrests (RR 0.60, 95\% CI 0.32 to 1.14) (Kinlock et al., 2005). Three studies (472 participants) showed no significant reduction in reincarceration (RR 0.77, 95\% CI 0.36 to 1.64) (Dolan et al., 2003, Dole et al., 1969, Kinlock et al., 2005) and one study (51 participants) showed a significant reduction in criminal activity (MD $-74.21,95 \%$ CI -133.53 to -14.89 ) (Kinlock et al., 2005).

\section{Agonist (methadone) versus partial agonist (buprenorphine)}

Two studies (370 participants) showed no significant reduction in self-reported drug use (RR 1.04, 95\% CI 0.69 to 1.55) (Magura et al., 2009, Wright et al., 2011). One study (81 participants) showed no significant reduction in self-reported drug use (MD $0.70,95 \%$ CI -5.33 to 6.73 ) (Magura et al., 2009). One study (116 participants) showed no significant reduction in criminal activity (RR $1.25,95 \%$ CI 0.83 to 1.88 ) (Magura et al., 2009).

\section{Agonist (methadone) versus antagonist (naltrexone)}

A single study (44 participants) showed no significant reduction in self-reported drug use (MD 4.60, 95\% CI -3.54 to 12.74 ) and showed no significant reduction in dichotomous (RR $1.10,95 \%$ CI 0.37 to 3.26 ) or continuous measures of reincarceration (MD $-0.50,95 \% \mathrm{CI}-8.04$ to 7.04 ) (Lobmaier et al., 2010).

\section{Agonist (methadone) and non-pharmacological intervention versus non-pharmacological intervention}

Two studies (203 participants) showed no significant reduction in self-reported drug use (RR 0.39, 95\% CI 0.14 to 1.13) (Bayanzadeh, 2004, Kinlock et al., 2007).

\section{Agonist (vigabatrin) versus placebo}

One study (103 participants) showed no evidence of a difference in terms of the number of negative urine tests for cocaine (MD 2.1, 95\% CI - 0.34 to 4.54) and end of trial abstinence for marijuana (RR $0.64,95 \%$ CI 0.16 to 2.52 ) but showed evidence of a difference in the end of trial abstinence (RR $1.06,95 \%$ CI 0.28 to 4.01 ) and 4-week abstinence for cocaine (RR 6.36, 95\% CI 1.50 to 27.01) (Bayanzadeh, 2004, Brodie et al., 2009, Kinlock et al., 2007).

\section{Antagonist (naloxone) versus no intervention (placebo or waiting list control)}

Three studies (333 participants) showed no significant reduction in self-reported drug use (RR 1.06, 95\% CI 0.87 to 1.28) (Hanlon et al., 1975, Hanlon et al., 1977, Parmar et al., 2017). 


\section{a}

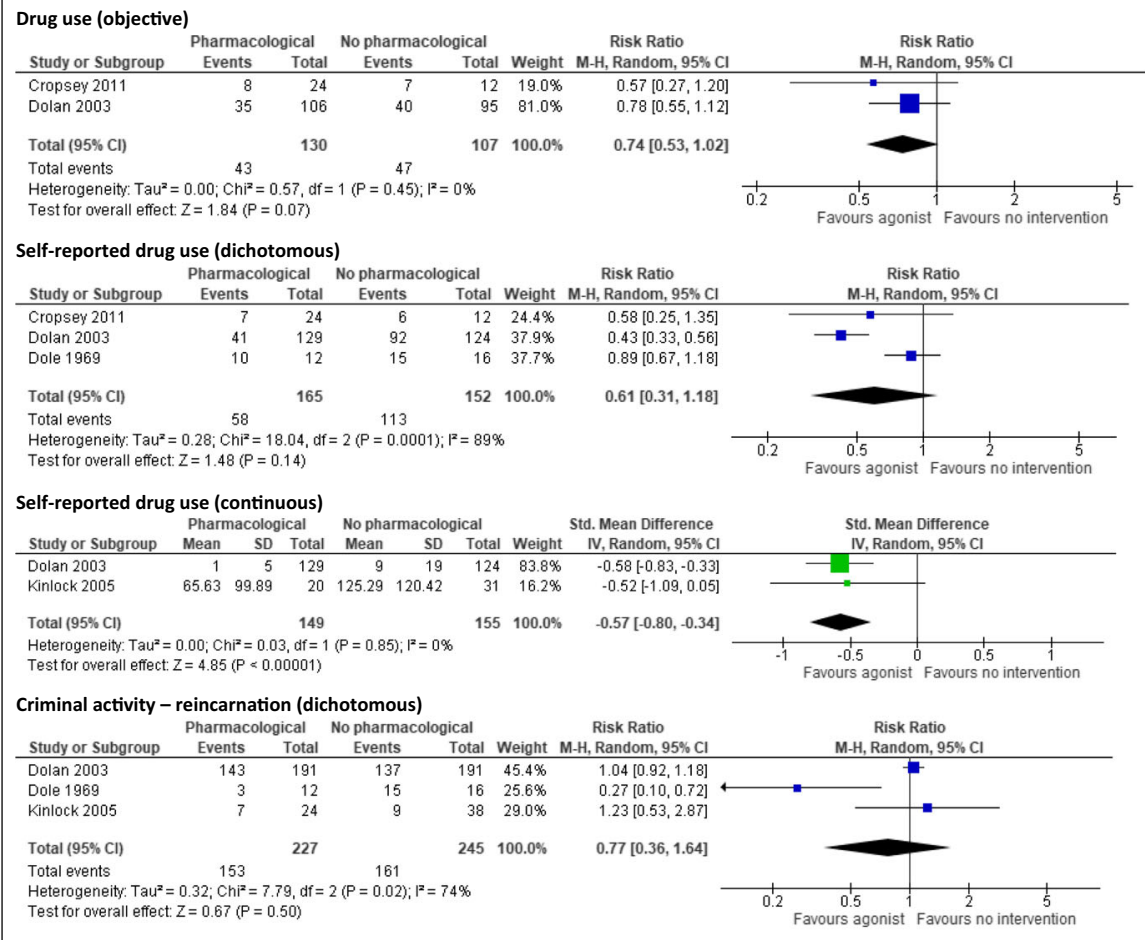

\section{b}

Self-reported drug use (dichotomous)

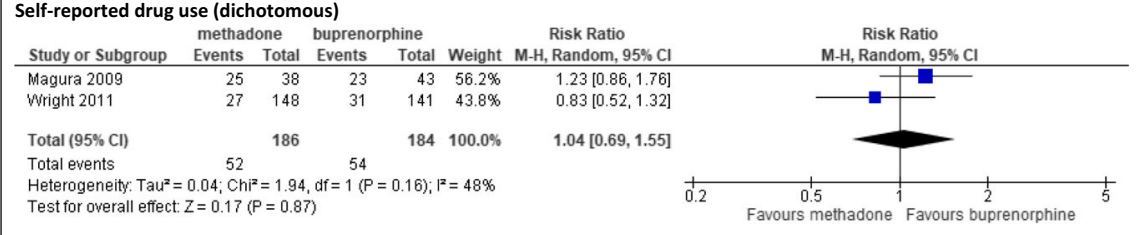

\section{C}

Self-reported drug use (dichotomous)

\begin{tabular}{|c|c|c|c|c|c|c|c|c|c|}
\hline \multirow{2}{*}{ Study or Subgroup } & \multicolumn{2}{|c|}{ Experimental } & \multicolumn{2}{|c|}{ Control } & \multirow{2}{*}{ Weight } & Risk Ratio & \multirow{2}{*}{\multicolumn{2}{|c|}{$\begin{array}{c}\text { Risk Ratio } \\
\text { M-H, Random, } 95 \% \mathrm{Cl}\end{array}$}} & \\
\hline & Events & $\frac{10 t a l}{20}$ & Events & $\frac{10 t a l}{21}$ & & M-H, Ranuom, 95\% CI & & & \\
\hline Kinlock 2007 & 16 & 70 & 22 & 64 & $51.0 \%$ & $0.66[0.38,1.15]$ & & & \\
\hline Total $(95 \% \mathrm{Cl})$ & & 108 & & 95 & $100.0 \%$ & $0.39[0.14,1.13]$ & & & \\
\hline Total events & 24 & & 51 & & & & & & \\
\hline $\begin{array}{l}\text { Heterogeneity: Tau² } \\
\text { Test for owerall effec }\end{array}$ & $\begin{array}{l}0.50 ; \mathrm{Ch} \\
Z=1.73\end{array}$ & $\begin{array}{l}=6.56, \\
=0.08\end{array}$ & $d f=1(P$ & $=0.01)$ & $\left.\right|^{2}=85 \%$ & & $\begin{array}{cc}1 & 1 \\
\text { Favours }^{\prime} & 0.5 \\
& 1\end{array}$ & $\begin{array}{c}2 \\
2 \\
\text { Favours NP }\end{array}$ & 5 \\
\hline
\end{tabular}

Fig. 3 Forest plots of intervention effects

\section{Antagonist (naltrexone) versus non-pharmacological treatment}

Two studies (371 participants) showed a significant reduction in subsequent opioid use (RR $0.60,95 \%$ CI 0.44 to 0.81) (Coviello et al., 2010, Lee et al., 2016). Two studies 


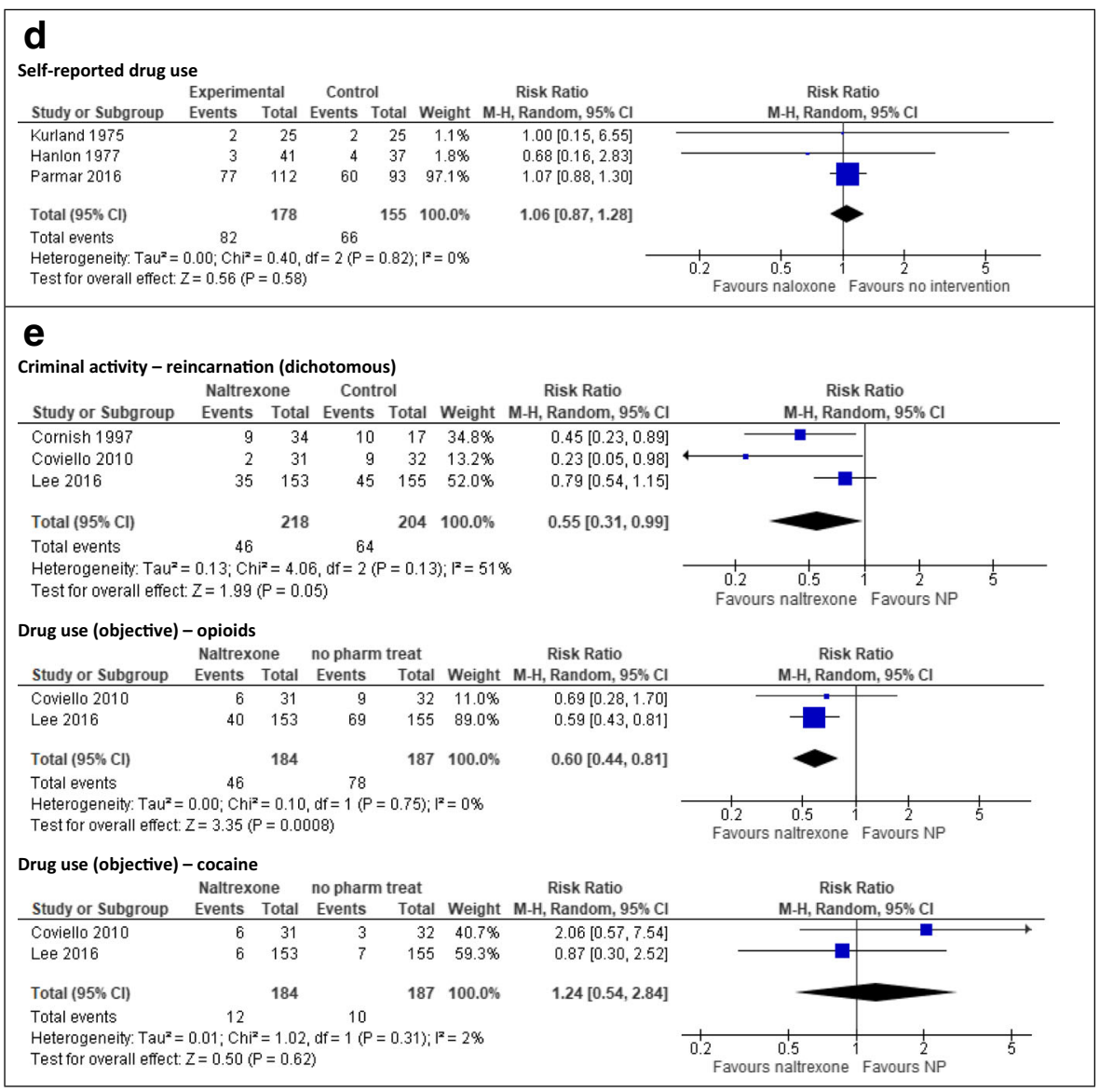

Fig. 3 continued.

(371 participants) showed no significant reduction in cocaine use (RR 1.24, 95\% CI 0.54 to 2.84) (Coviello et al., 2010, Lee et al., 2016). Three studies (422 participants) showed a significant reduction in subsequent reincarceration (RR $0.55,95 \% \mathrm{CI} 0.31$ to 0.99) (Cornish et al., 1997, Coviello et al., 2010, Lee et al., 2016). One study (63 participants) showed no significant reduction in subsequent drug charges (RR 3.10, 95\% CI 0.34 to 28.19) (Coviello et al., 2010).

\section{Mixed antagonist and agonist (cyclazocine) versus antagonist (naloxone)}

One study (70 participants) showed no significant reduction in abstinence at 6 months comparing mixed antagonist and agonist (cyclazocine) with the antagonist naloxone (RR 1.14, 95\% CI 0.46 to 2.81) (Hanlon et al., 1975). 


\section{Agonist (methadone) versus forced tapered withdrawal from methadone}

One study (283 participants) explored continued use of methadone within a prison setting compared with forced tapered withdrawal of methadone (Rich et al., 2015). The study found no significant difference in reduction in any drug use at 1 month following release from prison (RR $1.05,95 \%$ CI 0.83 to 1.34), opioid use at 1 month following release (RR $0.56,95 \%$ CI 0.26 to 1.22 ) or use of injectable illegal drugs (RR 0.67, 95\% CI 0.40 to 1.15 ) (Rich et al., 2015). The study also found no difference between the two options in terms of reincarceration at 1 month post release from prison (RR 1.49, 95\% CI 0.63 to 3.53 ) (Rich et al., 2015).

\section{Agonist (methadone) prior to release with financial support versus agonist (methadone) on release with no financial support}

One study (46 participants) explored the impact of providing financial support for methadone treatment following release from prison compared with no financial support on drug outcomes and criminal justice outcomes (McKenzie et al., 2012). The study found no significant difference between the two options in terms of heroin use in the past 30 days (RR $0.46,96 \%$ CI 0.20 to 1.03 ), use of other opiates in the past 30 days (RR $0.12,95 \%$ CI 0.01 to 2.22), use of crack/cocaine in the past 30 days (RR 0.46, 95\% CI 0.20 to 1.03 ), marijuana use in the past 30 days (RR $0.42,95 \%$ CI 0.09 to 2.07), polydrug use in the past 30 days (RR $0.70,95 \%$ CI 0.38 to 1.28 ) or any drug injecting in the past 30 days (RR $0.32,95 \%$ CI 0.10 to 1.04) (McKenzie et al., 2012). The study reported no significant difference between the interventions for reincarceration (RR $0.42,95 \%$ CI $0.09,2.07$ ) or arrest within the past 6 months (RR $1.26,95 \%$ CI 0.54, 2.96) (McKenzie et al., 2012).

\section{Narrative findings}

Narrative findings were presented for three studies where suitable data for extraction and exclusion in the meta-analysis were not available. One study compared methylphenidate or placebo to a non-pharmacological intervention (Konstenius et al., 2014). In this study, male participants with attention deficit disorder receiving methylphenidate had a higher proportion of drug negative urine test results compared with the placebo group $(p=0.047)$. A second study compared a specialist facility for treatment of methadone versus primary care plus suboxone (buprenorphine and naloxone) versus a specialist facility for suboxone treatment (Brown et al., 2013). At 6 months, the authors reported no use of opioids in any of the randomised groups (total of 15 participants). The final study compared methadone and placebo versus lofexidine and placebo (Howells et al., 2002). The authors reported no statistical reduction in the amount $(p=0.36)$ or intensity $(p=0.46)$ of opioid (heroin) use in the last month.

\section{Adverse events}

Seven of the $22(32 \%)$ studies did not report any information on adverse events, side effects of drugs nor referred to drug safety (Bayanzadeh, 2004, Brown et al., 2013, Dolan et al., 2003, Dole et al., 1969, Hanlon et al., 1975, Hanlon et al., 1977, Kurland 
et al., 1975). Three $(3 / 22,14 \%)$ studies measured adverse events, but did not report the findings (Coviello et al., 2010, Cropsey et al., 2011, Wright et al., 2011). Forty-one percent of the studies (9/22) reported only mild or moderate adverse events (Brodie et al., 2009, Cornish et al., 1997, Howells et al., 2002, Kinlock et al., 2005, Konstenius et al., 2014, Lee et al., 2016, Lobmaier et al., 2010, Magura et al., 2009, Parmar et al., 2017). One study reported 10 serious adverse events including nine hospitalisations (Kinlock et al., 2007). The hospitalisations included two cases for heart disease and one each for pneumonia, alcohol detoxification, kidney disease, high blood pressure, psychiatric problems and back pain (Kinlock et al., 2007). Two studies reported a fatal overdose (Kinlock et al., 2007, Rich et al., 2015) and one study reported two fatal overdoses (McKenzie et al., 2012).

\section{Economic outcomes}

The economic appraisal using the Drummond criteria identified four studies reporting some cost information (Magura et al., 2009, Murphy et al., 2017, Rich et al., 2015, Warren et al., 2006). Table 4 shows the results of the type of resource costs described for each paper. Three of the four studies were full economic evaluations reporting both incremental costs and outcomes (Magura et al., 2009, Murphy et al., 2017, Warren et al., 2006).

In terms of the overall Drummond classification (Drummond et al., 2005), three studies were coded as 4 (a full economic evaluation) and one study was coded as 3 (partial economic evaluation).

The Magura 2009 (Magura et al., 2009) study noted differences in the costs of administering buprenorphine and methadone, but were not sufficient for us to conduct a full cost-effectiveness appraisal. The investigators estimated that about ten times as many inmates can be served with methadone as with buprenorphine with the same staff resources. This cost implication is also endorsed in the community, where physicians have difficulty in obtaining reimbursement for buprenorphine treatment for released inmates, making the continued use of buprenorphine problematic after release.

The Murphy 2017 (Murphy et al., 2017) study conducted a full economic analysis reporting incremental cost-effectiveness ratios (ICERs), costs, QALYs and abstinent years of using extended-release naltrexone (XR-NTX) compared with treatment as usual (TAU) at 25 weeks and 78 weeks, respectively. The 25-week ICERs were reported as $\$ 162,150 / \mathrm{QALY}$ and $\$ 46,329$ / abstinent year. The ICER values at 78 weeks were \$76,400/QALY and \$16,371/abstinent year. The study reported that XR-NTX was effective in increasing both QALYs and abstinence rates compared with TAU. However, it was not cost-effective at the normally accepted threshold levels in the US, primarily due to the high price of the injection.

The Warren 2006 (Warren et al., 2006) study estimated the cost-effectiveness of the New South Wales (NSW) prison methadone programme compared with no methadone program. The study reported the annual cost of providing prison methadone in NSW as AUD \$2.9million (or AUD\$3234 per inmate per year) and the ICER as AUD \$38 per additional heroin-free day. It also reported that prison methadone is not costlier than community methadone, and it reduces morbidity and mortality through decreasing heroin use in prisons. 


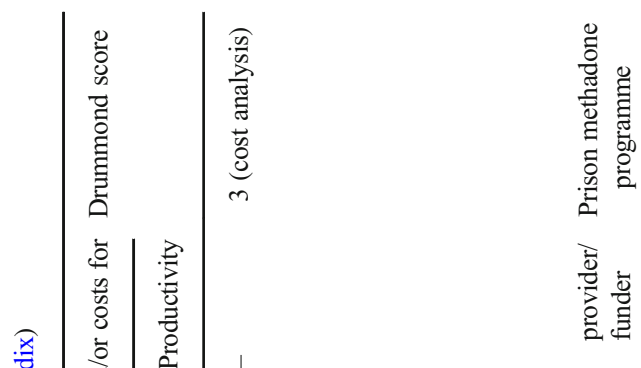

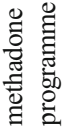

量

ठే

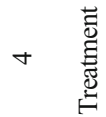

莺

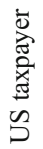

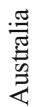

焉

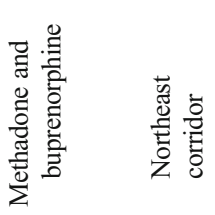

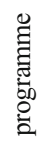

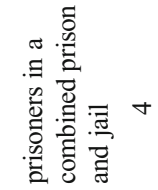

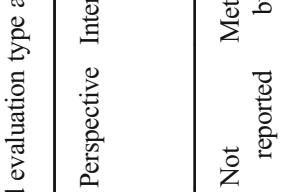

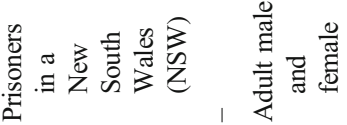

吉

ฮี ๙

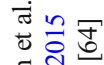

品

$>\left.\right|^{3}, \frac{\pi}{\sqrt[n]{n}}$

(a)

:ี

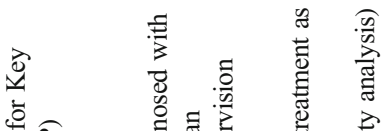

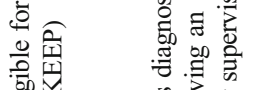

家近

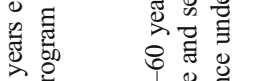



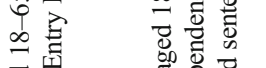

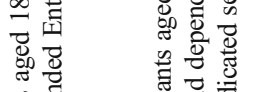

娄

:

余 言

节证

武焉递

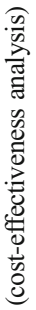

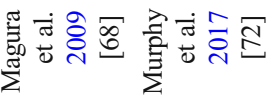

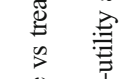

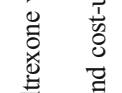

जे
0
0
0
0 
The final study by Rich 2015 (Rich et al., 2015) reported, as a secondary outcome, on healthcare costs (per inmate) associated with continued methadone maintenancetreatment programme compared with usual care (i.e. forced withdrawal from methadone) at 1 month after release from incarceration. Continued methadone treatment resulted in higher (methadone) treatment cost per individual compared with forced withdrawal ( $\$ 362$ vs $\$ 225, p<0.001$ ). The additional treatment costs were offset by savings incurred by reduced cost for physician ( $\$ 6.60 \mathrm{vs} \$ 8.80, p=0.793)$ and medical care ( $\$ 211$ vs $\$ 372, p=0.894$ ) after release, resulting in lower total costs ( $\$ 609$ vs $\$ 637, p<0.001)$. Continued methadone treatment also resulted in a greater probability of attendance at a methadone clinic after release and was more effective than forced withdrawal.

\section{Risk of bias assessment}

Although all the included studies were randomised trials, the level of reporting was uneven and many had unclear or high risk of bias for at least one element of the risk of bias assessment. Nearly $50 \%$ were rated as unclear risk of bias in terms of random sequence generation and over $50 \%$ of the trials were rated as unclear in terms of allocation concealment. $32 \%(7 / 22)$ of the studies were rated as low risk of bias for participant and personnel blinding and $82 \%(18 / 22)$ were rated as unclear on both subjective and objective measures of detection bias. Over $75 \%$ of the trials were at high risk of bias because they did not report all of the outcome data they set out to collect.

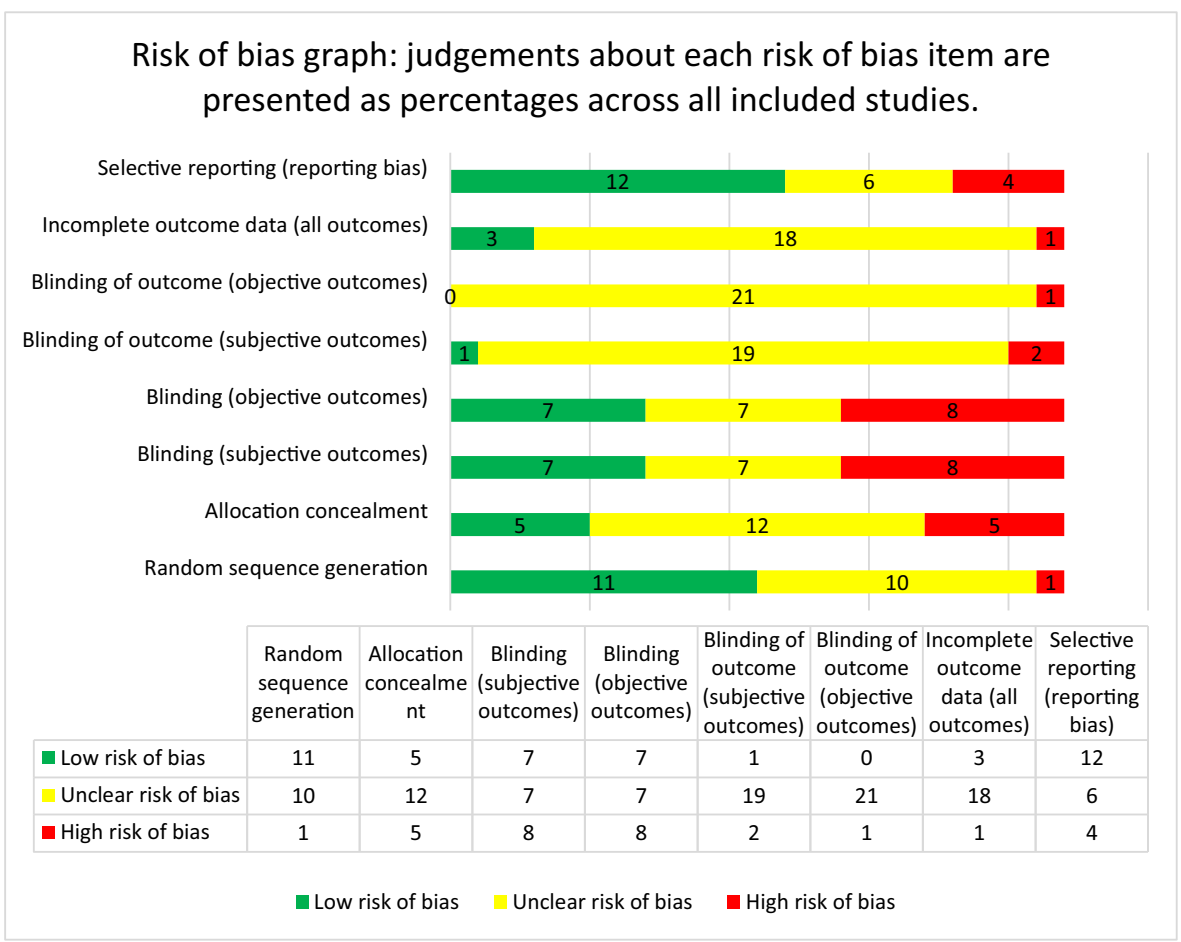

Fig. 4 Risk of bias judgements 
Over $50 \%$ of the studies were rated at low risk for selective reporting. The overall risk of bias judgements of the included studies are shown in Fig. 4, and summary and detailed accounts of the risk of bias assessment can be found in the Appendix.

\section{Conclusions}

The results of this systematic review provide some evidence to answer our original review questions. The review identified that we have some evidence to suggest that pharmacological interventions are effective in reducing drug use and criminal activity (Perry et al., 2015). The updated review revealed a total of 22 trials involving 4372 participants. The 22 trials compared pharmacological interventions or compared pharmacological interventions to no intervention and/or a placebo. Most studies were conducted in the USA, with the majority involving male adult offenders. The updated meta-analyses included 19/22 quantitative studies. Ten different pharmacological drugs were evaluated including methadone, buprenorphine, naltrexone, methylphenidate, vigabatrin, suboxone, naloxone, lofexifine, naltrexone and cyclazocine. Methadone in comparison with no treatment was the only comparison to demonstrate a significant reduction in subsequent drug use (using biological measures), self-reported drug use (using continuous measures) and criminal activity. When compared with methadone, no significant differences were found for buprenorphine, naltrexone or suboxone. None of these studies identified a significant reduction in either criminal activity and/or drug use. One study comparing buprenorphine to placebo (Cropsey et al., 2011) was the only study to involve wholly female offenders. More pharmacological trials of female offenders are required to identify comparability between men and women.

Studies of naloxone and methylphenidate in comparison with a placebo and nonpharmacological intervention, respectively, showed no significant differences for selfreported drug use. The evidence supporting the use of naloxone was explored using two very old studies (Hanlon et al., 1977, Hanlon et al., 1975) and one more recent study that had to be halted early due to problems with the randomisation procedure (Parmar et al., 2017). Naltrexone in comparison with a non-pharmacological intervention showed a significant reduction in self-reported drug use and reincarceration. The use of extended naltrexone pre and post prison release has produced some promising results, finding no overdoses during the treatment process (Lee et al., 2016). This study also reported that use of extended-release naltrexone was associated with a rate of opioid relapse that was lower than that with usual treatment at 24 weeks, but later comparisons (at 52 and 78 weeks) found no difference between the use of extendedrelease naltrexone and use of a brief counselling intervention (Lee et al., 2016). One study investigated methadone continuation treatment compared with forced tapered withdrawal from methadone within a prison setting, but found no significant difference in the reduction of any drug use at 1 month following release (Rich et al., 2015). The study also found no difference between the treatment options where reincarceration occurred at 1 month post release from prison. The impact of providing financial support for methadone treatment following release from prison was compared with no financial support in one study, but no significant difference was observed in terms of drug use or criminal justice outcomes (McKenzie et al., 2012). The study also reported no significant difference between the interventions in relation to arrest or reincarceration at six- 
month follow-up. We were unable to answer our third review questions about the impact of treatment setting on drug use or criminal activity. This was primarily because of the heterogeneity of the study comparisons and outcome measures. Given the relatively scarce resources for drug treatment evaluation opportunities in this population, future research should seek to build on existing trial data to develop an evidencebase to allow for such comparisons to be made.

The majority of studies included in the review reported only mild to moderate adverse effects, or did not report on drug safety as part of the trial information. One study reported serious adverse effects and three studies reported overdoses. Adverse effects should be measured consistently across any pharmacological drug trial to capture important data about drug safety. The current evidence does not provide a comprehensive overview of drug safety within this population.

Our final review question focused on the comparative costs and cost-effectiveness evidence of using such interventions to reduce drug use and criminal activity. An appraisal of the economic evidence identified studies with costs and outcomes data for pharmacological treatments among drug-using offenders in three studies and costs data in one study. All studies reported data on the direct medical costs associated with the provision of treatment (Magura et al., 2009, Murphy et al., 2017, Rich et al., 2015, Warren et al., 2006) and one study also included costs related to criminal justice (Murphy et al., 2017). The findings from the studies revealed the higher effectiveness of pharmacological treatments in terms of increased QALYs and abstinent years (Murphy et al., 2017) and reduced heroin use (Warren et al., 2006). One study reported methadone to be cost-effective at the current willingness to pay thresholds (Warren et al., 2006). Another study reported continued methadone treatment to dominate forced withdrawal in terms of costs and effects (Rich et al., 2015). More research is needed to compare the relative cost-effectiveness of a range of treatments (pharmacotherapies, non-pharmacological interventions and combined treatments) in prison and community-based criminal justice settings.

Additionally, the studies contained mainly male offenders, with only one study containing a $100 \%$ sample of female offenders. The majority of studies were conducted in the USA. Together these factors limit the external validity and generalisability of the study findings. For these reasons, the studies are unlikely to reflect different international practice in the criminal justice system. Besides the limitations already discussed, the search methodology was limited to databases that could be accessed via the University of York and extensive website searches were not conducted. As a result, some literature may have been missed from this updated version. Specific search terms were not used to identify cost and cost-effectiveness studies in 3/12 databases which meant that some economic studies might have been missed from this review.

Several research implications can be identified from this review. Generally, better quality research is required to evaluate the effectiveness of interventions with extended long-term effects of aftercare following release into the community. Buprenorphine research in the prison environment requires evidence of the long-term impact and larger studies; currently, an equivalence of buprenorphine and methadone exists. Future clinical trials should collect information from all sectors of the criminal justice system. This would enhance the heterogeneous nature of the included studies and would facilitate generalisation of study findings. Evidence of comparable mortality rates in prisoners using pharmacological interventions (particularly after release) needs to be 
explored to assess the long-term outcomes of such treatments, perhaps using alternative study designs to randomised controlled trials. The link between dosage, treatment retention and subsequent criminal activity should be examined across all three pharmacological treatment options. Evidence from other trial data suggests that dose has important implications for retention in treatment; in future studies, this should be considered alongside criminal activity outcomes. Cost and cost-effectiveness information should be standardised within trial evaluations; this will help policymakers to decide upon health versus criminal justice costs.

Acknowledgements We would like to acknowledge the help of the York Health Economics Consortium (YHEC), the Department of Health Sciences at the University of York and the Cochrane Drugs and Alcohol Group.

Author contributions Amanda E. Perry, Caroline Pearson, Aboaja Anne, Thakkar Pratish, Santosh Kumar, Lucy Burns, Marrissa Martyn-St James and Julie Glanville all contributed to the screening of abstracts for inclusion and extraction of data for the final report. Kath Wright conducted and advised on the updated search strategies. Matthew Bunney supported the presentation and summary of data for the report. Catherine Hewitt provided statistical expertise on the meta-analyses and Shilpi Swarmi conducted the appraisal of economic evidence.

\section{Compliance with ethical standards}

Conflict of interest The authors declare that they have no conflict of interest.

\section{Appendix}

\section{Search Strategy}

The searches for this updated review were conducted in February 2019 in the following databases:

ASSIA, CENTRAL, CINAHL, Criminal Justice Abstracts, Embase, HMIC, LILACS, MEDLINE, PAIS, PsycINFO, Science Citation Index, Social Science Citation Index.

Initially, there were 4384 records identified. After deduplication, there were 2603 records remaining. After further deduplication against the results of the January 2018 update search, 2445 records remained.

\subsection{ASSIA search strategy}

\footnotetext{
ASSIA via ProQuest search strategy (search date $=$ 6th February 2019, 247 records identified)

(ti(substance* NEAR/2 (addict* OR depend* OR disorder* OR abuse* OR abusing OR misuse* OR misusing OR consumption* OR withdraw* OR withdraw* OR detox*)) OR ab(substance* NEAR/2 (addict* OR depend* OR disorder* OR abuse* OR abusing OR misuse* OR misusing OR consumption* OR withdraw* OR withdraw* OR detox*)) OR ti(drug* NEAR/2 (addict* OR depend* OR disorder* OR abuse* OR abusing OR misuse* OR misusing OR consumption* OR withdraw* OR withdraw* OR
} 
detox*)) OR ab(drug* NEAR/2 (addict* OR depend* OR disorder* OR abuse* OR abusing OR misuse* OR misusing OR consumption* OR withdraw* OR withdraw* OR detox*)) OR ti(narcotic* NEAR/2 (addict* OR depend* OR disorder* OR abuse* OR abusing OR misuse* OR misusing OR consumption* OR withdraw* OR withdraw* OR detox*)) OR ab(narcotic* NEAR/2 (addict* OR depend* OR disorder* OR abuse* OR abusing OR misuse* OR misusing OR consumption* OR withdraw* OR withdraw* OR detox*)) OR ti(mdma OR alcohol* OR opiate* OR opioid* OR opium OR heroin OR methadone OR cocaine OR amphetamine* OR marijuana OR cannabis OR crack OR phencyclidine) OR ab(mdma OR alcohol* OR opiate* OR opioid* OR opium OR heroin OR methadone OR cocaine OR amphetamine* OR marijuana OR cannabis OR crack OR phencyclidine)) AND (ti(justice system) OR remand* OR parole* OR probation OR court* OR corrections OR correctional OR revocation) OR ab((justice system) OR remand* OR parole* OR probation OR court* OR corrections OR correctional OR revocation) OR ti(crime OR offend* OR criminal OR convict* OR felon*) OR ab(crime OR offend* OR criminal* OR convict* OR felon*) OR ti(custody OR custodial OR gaol* OR jail* OR prison* OR incarcerat* OR inmate*) OR ab(custody OR custodial OR gaol* OR jail* OR prison* OR incarcerat*or inmate*) OR ti(reoffend* OR reincarcerat* OR recidiv* OR ex-offender*) OR ab(reoffend* OR reincarcerat* OR recidiv* OR ex-offender*)) AND pd(20180101-20190201)

\subsection{The CENTRAL Register of Controlled Trials search strategy}

\section{CENTRAL via Cochrane Library search strategy (search date $=6$ th February 2019, 78 records identified)}

\#1MeSH descriptor: [Substance-Related Disorders] explode all trees

\#2MeSH descriptor: [Illicit Drugs] explode all trees

\#3MeSH descriptor: [Designer Drugs] explode all trees

\#4MeSH descriptor: [Narcotics] explode all trees

\#5(substance* or drug* or narcotic*) near/2 (addict* or depend* or disorder* or abuse* or abusing or misuse* or misusing or consumption* or withdraw\$ or withdraw* or detox*):ti,ab,kw (Word variations have been searched)

\#6mdma or alcohol* or opiate* or opioid* or opium or heroin or methadone or cocaine or amphetamine* or marijuana or cannabis or crack or phencyclidine:ti,ab,kw (Word variations have been searched)

$\# 7 \# 1$ or \#2 or \#3 or \#4 or \#5 or \#6

\#8MeSH descriptor: [Crime] explode all trees

\#9MeSH descriptor: [Criminals] explode all trees

\#10MeSH descriptor: [Prisoners] explode all trees

\#11(justice system) or remand* or parole* or probation or court* or corrections or correctional or revocation:ti,ab,kw (Word variations have been searched)

$\# 12$ custody or custodial or gaol* or jail* or prison* or incarcerat* or inmate*:ti,ab,kw (Word variations have been searched)

\#13reoffend* ${ }^{*}$ or reincarcerat* or recidiv* or ex-offender*:ti,ab,kw (Word variations have been searched)

$\# 14$ offend* or criminal* or convict* or felon:ti,ab,kw (Word variations have been searched)

$\# 15 \# 8$ or \#9 or \#10 or \#11 or \#12 or \#13 or \#14

$\# 16 \# 7$ and \#15

\subsection{CINHAL Plus search strategy}

CINHAL Plus via EBSCO search strategy (search date $=$ 6th February 2019, 774 records identified) 
TI (substance* N2 (addict* or depend* or disorder* or abuse* or abusing or misuse* or misusing or consumption* or withdraw* or withdraw* or detox*)) OR AB (substance* N2 (addict* or depend* or disorder* or abuse* or abusing or misuse* or misusing or consumption* or withdraw* or withdraw* or detox*)) OR TI (drug* N2 (addict* or depend* or disorder* or abuse* or abusing or misuse* or misusing or consumption* or withdraw* or withdraw* or detox*)) OR AB (drug* N2 (addict* or depend* or disorder* or abuse* or abusing or misuse* or misusing or consumption* or withdraw* or withdraw* or detox*)) OR TI (narcotic* N2 (addict* or depend* or disorder* or abuse* or abusing or misuse* or misusing or consumption* or withdraw* or withdraw* or detox*)) OR AB (narcotic* N2 (addict* or depend* or disorder* or abuse* or abusing or misuse* or misusing or consumption* or withdraw* or withdraw* or detox*))

S2 TI (mdma or alcohol* or opiate* or opioid* or opium or heroin or methadone or cocaine or amphetamine* or marijuana or cannabis or crack or phencyclidine) OR AB (mdma or alcohol* or opiate* or opioid* or opium or heroin or methadone or cocaine or amphetamine* or marijuana or cannabis or crack or phencyclidine)

\section{S3 S1 OR S2}

S4 TI (justice system) or crime or remand* or parole* or probation or court* or corrections or correctional or revocation) $\mathrm{OR} \mathrm{AB}$ (justice system) or crime or remand* or parole* or probation or court* or corrections or correctional or revocation) OR TI (offend* or criminal* or convict* or felon*) OR AB (offend* or criminal* or convict* or felon*) OR TI (custody or custodial or gaol* or jail* or prison* or incarcerat* or inmate*) OR AB (custody or custodial or gaol* or jail* or prison* or incarcerat* or inmate*) OR TI (reoffend* or reincarcerat* or recidiv* or ex-offender*) OR AB (reoffend* or reincarcerat* or recidiv* or ex-offender*)

\subsection{Criminal Justice Abstracts search strategy}

\section{Criminal justice abstracts via ProQuest search strategy (search date $=$ 6th February 2019, 594 records)}

S1 ab(reoffend* OR reincarcerat* OR recidiv* OR ex-offender*))

S2 ti(reoffend* OR reincarcerat* OR recidiv* OR ex-offender*) OR

S3 ab(custody OR custodial OR gaol* OR jail* OR prison* OR incarcerat*or inmate*) OR

S4 ti(custody OR custodial OR gaol* OR jail* OR prison* OR incarcerat* OR inmate*) OR

S5 ab(crime OR offend* OR criminal* OR convict* OR felon*) OR

S6 ti(crime OR offend* OR criminal OR convict* OR felon*) OR

S7 ab((justice system) OR remand* OR parole* OR probation OR court* OR corrections OR correctional OR revocation) $\mathrm{OR}$

S8 (ti((justice system) OR remand* OR parole* OR probation OR court* OR corrections OR correctional OR revocation) OR

$\mathrm{S} 9 \mathrm{~s} 1$ or $\mathrm{s} 2$ or $\mathrm{s} 3$ or $\mathrm{s} 4$ or $\mathrm{s} 5$ or $\mathrm{s} 6$ or $\mathrm{s} 7$ or $\mathrm{s} 8$

S10 ab(mdma OR alcohol* OR opiate* OR opioid* OR opium OR heroin OR methadone OR cocaine OR amphetamine* OR marijuana OR cannabis OR crack OR phencyclidine))

S11 ti(mdma OR alcohol* OR opiate* OR opioid* OR opium OR heroin OR methadone OR cocaine OR amphetamine* OR marijuana OR cannabis OR crack OR phencyclidine) OR

S12 ab(narcotic* NEAR/2 (addict* OR depend* OR disorder* OR abuse* OR abusing OR misuse* OR misusing OR consumption* OR withdraw* OR withdraw* OR detox*)) OR

S13 ti(narcotic* NEAR/2 (addict* OR depend* OR disorder* OR abuse* OR abusing OR misuse* OR misusing OR consumption* OR withdraw* OR withdraw* OR detox*)) OR 
ab(drug* NEAR/2 (addict* OR depend* OR disorder* OR abuse* OR abusing OR misuse* OR misusing OR consumption* OR withdraw* OR withdraw* OR detox*)) OR

S15 ti(drug* NEAR/2 (addict* OR depend* OR disorder* OR abuse* OR abusing OR misuse* OR misusing OR consumption* OR withdraw* OR withdraw* OR detox*)) OR

S16 ab(substance* NEAR/2 (addict* OR depend* OR disorder* OR abuse* OR abusing OR misuse* OR misusing OR consumption* OR withdraw* OR withdraw* OR detox*)) OR

S17 (ti(substance* NEAR/2 (addict* OR depend* OR disorder* OR abuse* OR abusing OR misuse* OR misusing OR consumption* OR withdraw* OR withdraw* OR detox*)) OR

$\mathrm{S} 18 \mathrm{~s} 10$ or $\mathrm{s} 11$ or $\mathrm{s} 12$ or $\mathrm{s} 13$ or $\mathrm{s} 14$ or $\mathrm{s} 15$ or $\mathrm{s} 16$ or $\mathrm{s} 17$

S19 s9 and s18

\subsection{EMBASE via Ovid search strategy}

\section{Embase via Ovid search strategy (search date $=6$ th February 2019, 213 records Database: Embase <2015 to 2019 Week 05)}

1 substance abuse/ (9875)

2 drug dependence/ (11329)

3 addiction/ (6500)

4 drug abuse/ (6705)

5 intravenous drug abuse/ (1611)

6 opiate addiction/ (5462)

7 heroin dependence/ (1491)

8 cocaine dependence/ (2564)

9 morphine addiction/ (586)

10 cannabis addiction/ (2161)

11 alcoholism/ (18489)

12 alcohol abuse/ (6835)

13 (substance\$ or drug \$ or narcotic\$) adj2 (addict\$ or depend\$ or disorder\$ or abuse \$ or abusing or misuse\$ or misusing or consumption $\$$ or withdraw\$ or withdraw\$ or detox\$)).ti,ab. (29221)

14 (mdma or alcohol\$ or opiate $\$$ or opioid $\$$ or opium or heroin or methadone or cocaine or amphetamine $\$$ or marijuana or cannabis or crack or phencyclidine).ti,ab. (146170)

151 or 2 or 3 or 4 or 5 or 6 or 7 or 8 or 9 or 10 or 11 or 12 or 13 or 14 (179011)

16 exp. crime/ (13956)

17 criminal behavior/ (1234)

18 criminal justice/ (1360)

19 prisoner/ or offender/ (6567)

20 (justice system or remand $\$$ or parole $\$$ or probation or court\$ or corrections or correctional or revocation).ti,ab. (13647)

21 (offend\$ or criminal\$ or convict\$ or felon\$).ti,ab. (10272)

22 (custody or custodial or gaol\$ or jail\$ or prison\$ or incarcerat\$ or inmate\$).ti,ab. (8080)

23 (reoffend\$ or reincarcerat\$ or recidiv\$ or ex-offender\$).ti,ab. (1723)

2416 or 17 or 18 or 19 or 20 or 21 or 22 or $23(40493)$

25 clinical trial/ (128414)

26 randomised controlled trial/ (169099)

27 randomisation/ (15096)

28 single blind procedure/ (14215)

29 double blind procedure/ (38131)

30 crossover procedure/ (15966)

31 placebo/ (71036)

32 randomi?ed. controlled trial\$.tw. (84975)

33 rct.tw. (14740) 
34 random allocation.tw. (482)

35 randomly allocated.tw. (9673)

36 allocated randomly.tw. (397)

37 (allocated adj2 random).tw. (83)

38 single blind\$.tw. (6643)

39 double blind\$.tw. (42229)

40 ((treble or triple) adj blind\$).tw. (480)

41 placebo\$.tw. (70811)

42 prospective study/ (221821)

43 or/25-42 (570137)

44 case study/ (27298)

45 case report.tw. (98676)

46 abstract report/ or letter/ (171138)

47 or/44-46 (293801)

4843 not 47 (558286)

4915 and 24 and 48 (516)

502018*.dc. (1740479)

512018*.dd. (832909)

522018*.dp. (200558)

532018*.em. (2210866)

54 "2018".yr. (1380865)

552019*.dc. (219266)

562019*.dd. (110044)

572019*.dp. (21703)

582019*.em. (436976)

59 "2019".yr. (138614)

6050 or 51 or 52 or 53 or 54 or 55 or 56 or 57 or 58 or 59 (2654076)

6149 and 60 (213)

\subsection{Health Management Information Consortium (HMIC) via Ovid search strategy}

\section{HMIC search strategy (sea date $=6$ th February 2019, 12 records}

$<1979$ to November 2018>)

1 designer drugs/ (6)

2 exp. narcotics/ (368)

3 ((substance $\$$ or drug\$ or narcotic\$) adj2 (addict\$ or depend $\$$ or disorder $\$$ or abuse $\$$ or abusing or misuse $\$$ or misusing or consumption $\$$ or withdraw $\$$ or withdraw $\$$ or detox $\$$ )).ti,ab. (3065)

4 (mdma or alcohol\$ or opiate $\$$ or opioid $\$$ or opium or heroin or methadone or cocaine or amphetamine\$ or marijuana or cannabis or crack or phencyclidine).ti,ab. (6988)

51 or 2 or 3 or $4(9104)$

6 crime/ (455)

7 prisoners/ (660)

8 (justice system or remand $\$$ or parole $\$$ or probation or court $\$$ or corrections or correctional or revocation).ti,ab. (3355)

9 (offend\$ or criminal\$ or convict\$ or felon\$).ti,ab. (2890)

10 (custody or custodial or gaol\$ or jail\$ or prison\$ or incarcerat\$ or inmate\$).ti,ab. (2360)

11 (reoffend $\$$ or reincarcerat $\$$ or recidiv $\$$ or ex-offender $\$$ ).ti,ab. (108)

126 or 7 or 8 or 9 or 10 or 11 (7182)

135 and 12 (642)

14 ("2018” or “2019”).yr. (1340)

1513 and 14 (1)

$16(2018 *$ or $2019 *)$.dp. (0)

$17\left(2018^{*}\right.$ or $\left.2019 *\right)$.up. (5221) 


\subsection{LILACS search strategy}

\section{LILACS search strategy (search date $=$ 6th February 2019, 15 records)}

tw:((remand or prison or prisoner or prisoners or prisäo or cárcere or cárcel or detenidos or detentas or acusados or presidiáriossobre or presidiarias or preso or Privados or recluses or offender\$ or infratoras or infratora or infratores or delicuentes or infrator or criminal $\$$ or probation or probatorio or estagio or court or courts or tribunal or tribunals or secure establishment\$ or secure facilit\$ or reoffend $\$$ or reincarcerat\$ or recidivi\$ or reincidencia or recidivante or reincidência or ex-offender\$ or jail or jails or gaol or gaols or incarcerat\$ or encarcerados or covict or convicts or convicted or felon or felons or conviction\$ or reconviction \$ or Convicçöes or convicciones or inmate\$ or internos or high security or prisoners or law enforcement or jurisprudence))) AND (tw:((Substance abuse\$ or substance misuse $\$$ or substance use $\$$ or usuários de substâncias or drug dependanc\$ or drug abuse $\$$ or drug use $\$$ or drug misuse $\$$ or drug addict\$ or narcotics addict\$ or narcotics use\$ or narcotics misuse\$ or narcotics abuse\$ or chemical dependenc\$ or opiates or heroin or crack or cocaine or amphetamines or cocaine or heroína or opioides or anfetaminas or opiáceos or opioids or addiction or adicción or adicciones or dependência or farmacodependente or adición or adiçäo or dependence disorder\$ or drug involved or Substance-related disorders or amphetamine-related disorders or

cocaine-related disorders or marijuana abuse or opioid-related disorders or phencyclidine abuse

\subsection{MEDLINE via Ovid search strategy}

\section{MEDLINE search strategy (search date=6th February 2019, 223 records}

Ovid MEDLINE(R) ALL < 1946 to February 04, 2019>)

1 exp. substance related disorders/ (263291)

2 street drugs/ (10346)

3 designer drugs/ (1476)

4 exp. narcotics/ (116718)

5 ((substance $\$$ or drug $\$$ or narcotic $\$$ ) adj2 (addict\$ or depend $\$$ or disorder $\$$ or abuse $\$$ or abusing or misuse $\$$ or misusing or consumption $\$$ or withdraw $\$$ or withdraw $\$$ or detox $\$)$ ).ti,ab. (96986)

6 (mdma or alcohol\$ or opiate\$ or opioid\$ or opium or heroin or methadone or cocaine or amphetamine\$ or marijuana or cannabis or crack or phencyclidine).ti,ab. (480977)

71 or 2 or 3 or 4 or 5 or $6(694112)$

8 crime/ (14858)

9 criminals/ (4255)

10 prisoners/ (15556)

11 (justice system or remand\$ or parole $\$$ or probation or court\$ or corrections or correctional or revocation).ti,ab. (55358)

12 (offend\$ or criminal\$ or convict\$ or felon\$).ti,ab. (36931)

13 (custody or custodial or gaol\$ or jail\$ or prison $\$$ or incarcerat $\$$ or inmate\$).ti,ab. (29129)

14 (reoffend $\$$ or reincarcerat $\$$ or recidiv\$ or ex-offender\$).ti,ab. (5486)

158 or 9 or 10 or 11 or 12 or 13 or $14(123890)$

167 and 15 (16320)

17 randomised controlled trial.pt. (475636)

18 controlled clinical trial.pt. (92894) 
19 randomised.ab. (433678)

20 placebo.ab. (195168)

21 drug therapy.fs. (2081276)

22 randomly.ab. (304772)

23 trial.ab. (452743)

24 groups.ab. (1877444)

2517 or 18 or 19 or 20 or 21 or 22 or 23 or 24 (4370157)

26 exp. animals/ not humans.sh. (4543138)

2725 not 26 (3778987)

2816 and 27 (3686)

$29(2018 *$ or $2019 *)$.dt. (1412794)

3028 and $29(183)$

31 (“2018” or “2019”).yr. (1444316)

3228 and 31 (202)

3330 or $32(223)$

\subsection{PAIS via ProQuest search strategy}

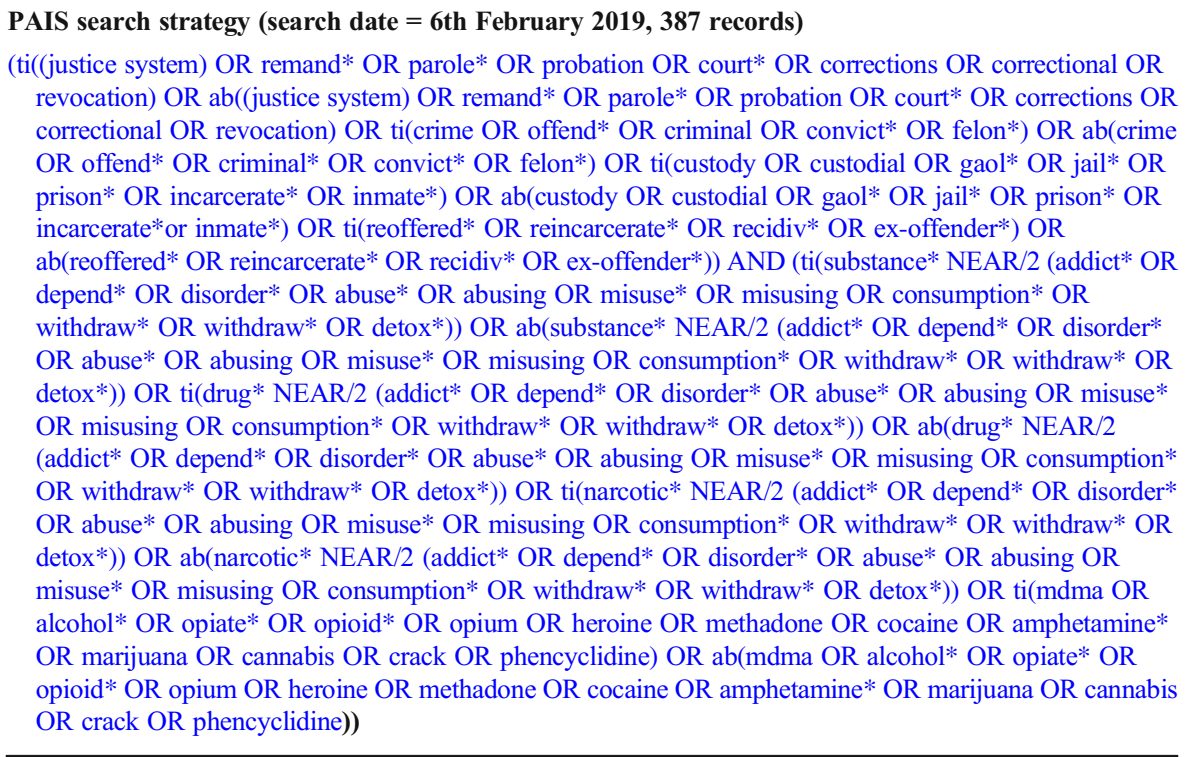

\subsection{PsycINFO via OVID search strategy}

\section{PsycINFO search strategy (search date=6th February 2019, 461 records Database: PsycINFO <1806 to January Week 4 2019>)}

1 Addiction/ (10001)

2 Drug dependency/ (12348) 
3 Drug Usage/ (17225)

4 Drug Abuse/ (45330)

5 Alcohol Abuse/ (17406)

6 Alcohol rehabiliation/ or drug rehabilitation/ (20161)

7 (substance $\$$ or drug $\$$ or narcotic\$) adj2 (addict $\$$ or depend $\$$ or disorder $\$$ or abuse $\$$ or abusing or misuse $\$$ or misusing or consumption\$ or withdraw\$ or withdraw\$ or detox\$)).ti,ab. (77627)

8 (mdma or alcohol\$ or opiate\$ or opioid $\$$ or opium or heroin or methadone or cocaine or amphetamine\$ or marijuana or cannabis or crack or phencyclidine).ti,ab. (184141)

91 or 2 or 3 or 4 or 5 or 6 or 7 or 8 (251016)

$10 \mathrm{crime} /(14495)$

11 criminal behavior/ (8666)

12 recidivism/ (5524)

13 prisoners/ or prisons/ or incarceration/ (17373)

$14 \mathrm{probation} /$ or parole/ (1918)

15 criminals/ or female criminals/ or male delinquency/ or juvenile delinquency/ (31452)

16 (justice system or remand $\$$ or parole $\$$ or probation or court $\$$ or corrections or correctional or revocation).ti,ab. (55660)

17 (offend\$ or criminal\$ or convict\$ or felon\$).ti,ab. (72593)

18 (custody or custodial or gaol\$ or jail\$ or prison\$ or incarcerat\$ or inmate\$).ti,ab. (38928)

19 (reoffend $\$$ or reincarcerat\$ or recidiv\$ or ex-offender\$).ti,ab. (8872)

2010 or 11 or 12 or 13 or 14 or 15 or 16 or 17 or 18 or $19(147687)$

21 (empirical study or treatment outcome clinical trial).md. (2342063)

22 (random\$ adj4 trial\$).ti,ab. (47794)

23 Placebo/ (5203)

24 (random* or sham or placebo*).ti,ab,hw. (214532)

25 ((singl* or doubl*) adj (blind* or dumm* or mask*)).ti,ab,hw. (24681)

2621 or 22 or 23 or 24 or 25 (2399281)

279 and 20 and 26 (11682)

282,018\$.up. (155517)

2927 and 28 (373)

30 "2018".dp. (142864)

3127 and $30(343)$

32 "2018".yr. (142864)

3327 and $32(343)$

3429 or 31 or $33(461)$

\subsection{Science Citation Index via Web of Science search strategy}

\section{Science Citation Index via Web of Science search strategy (search date $=$ 6th February 2019638 records Indexes = SCI-EXPANDED Timespan=1900-2019)}

\# 1 TOPIC: (substance* NEAR/2 (addict* or depend* or disorder* or abuse* or abusing or misuse* or misusing or consumption* or withdraw* or withdraw* or detox*) ) OR TOPIC: (drug* NEAR/2 (addict* or depend* or disorder* or abuse* or abusing or misuse* or misusing or consumption* or withdraw* or withdraw* or detox*) ) OR TOPIC:(narcotic* NEAR/2 (addict* or depend* or disorder* or abuse* or abusing or misuse* or misusing or consumption* or withdraw* or withdraw* or detox $\left.{ }^{*}\right)$ )

\# 2TOPIC: (mdma or alcohol* or opiate* or opioid* or opium or heroin or methadone or cocaine or amphetamine* or marijuana or cannabis or crack or phencyclidine)

\# 3 \#2 OR \#1

\# 4TOPIC: ("justice system" or remand* or parole* or probation or court* or corrections or correctional or revocation) OR TOPIC: (crime or criminal or offender* or criminal* or convict* or felon*) OR TOPIC: (custody or custodial or gaol* or jail* or prison* or incarcerat* or inmate*) OR TOPIC: (reoffend* or reincarcerat* ${ }^{*}$ or recidiv* or ex-offender*)

\#5\#4 AND \#2 


\subsection{Social Science Citation Index via Web of Science search strategy}

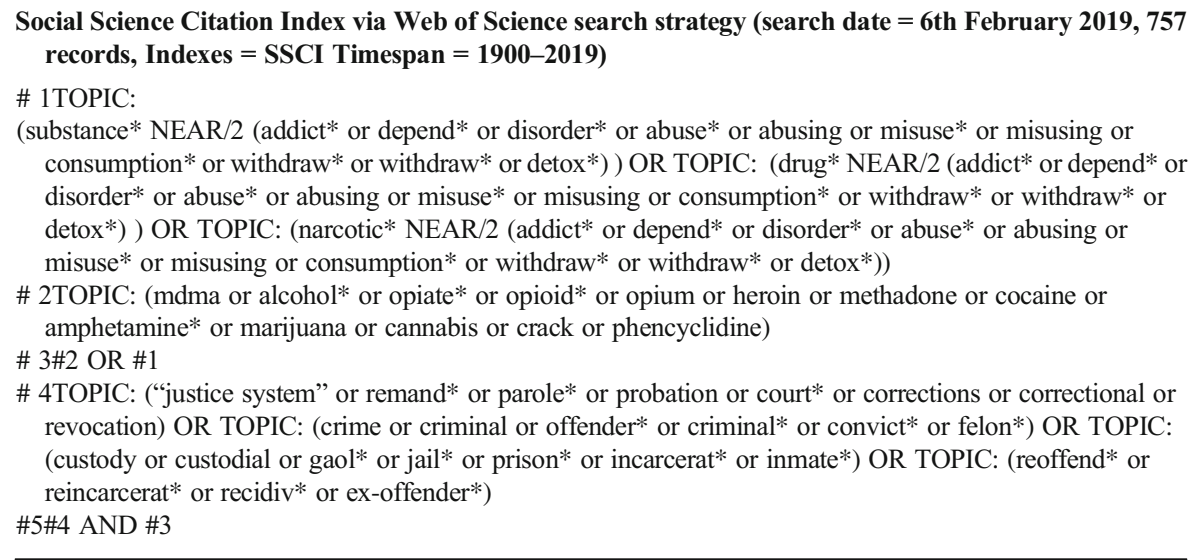

\section{Screening criteria}

The screening process was divided into two key phases. Phase one used seven key questions reported in the original new reference review:

\section{Prescreening criteria: phase one}

Is the document an empirical study? [If "no" exclude document.]

Does the study evaluate an intervention, a component of which is designed to reduce, eliminate or prevent relapse among drug-using offenders?

Are the participants referred by the criminal justice system at baseline?

Does the study report pre-programme and post-programme measures of drug use?

Does the study report pre-programme and post-programme measures of criminal behaviour?

Is the study a randomised controlled trial?

Do the outcome measures refer to the same length of follow-up for two groups?

Papers included after phase one screening were then scrutinised to assess:

\section{Prescreening: phase two}

Is the intervention a pharmacological intervention? [if "yes" include document] 


\section{Drummond (1996) checklist-risk of bias assessment tool for economic evaluations}

\section{Study question}

Study design

1. Was the research question stated?

2. Was the economic importance of the research question stated?

3. Was/were the viewpoint(s) of the analysis clearly stated and justified?

4. Was a rationale reported for the choice of the alternative programmes or interventions compared?

5. Were the alternatives being compared clearly described?

6. Was the form of economic evaluation stated?

7. Was the choice of form of economic evaluation justified in relation to the questions addressed?

Data collection

8. Was/were the source(s) of effectiveness estimates used stated?

9. Were details of the design and results of the effectiveness study given (if based on a single study)?

10. Were details of the methods of synthesis or meta-analysis of estimates given (if based on an overview of a number of effectiveness studies)?

11 . Were the primary outcome measure(s) for the economic evaluation clearly stated?

12. Were the methods used to value health states and other benefits stated?

13. Were the details of the subjects from whom valuations were obtained given?

14. Were productivity changes (if included) reported separately?

15 . Was the relevance of productivity changes to the study question discussed?

16. Were quantities of resources reported separately from their unit cost?

17. Were the methods for the estimation of quantities and unit costs described?

18. Were currency and price data recorded?

19. Were details of price adjustments for inflation or currency conversion given?

20. Were details of any model used given?

21. Was there a justification for the choice of model used and the key parameters on which it was based?

Analysis and interpretation of results

22. Was the time horizon of cost and benefits stated?

23. Was the discount rate stated?

24. Was the choice of rate justified?

25. Was an explanation given if cost or benefits were not discounted?

\section{Study name \\ Grade (yes/no/not Comments clear/N/A)}


26. Were the details of statistical test(s) and confidence intervals given for stochastic data?

27 . Was the approach to sensitivity analysis described?

28 . Was the choice of variables for sensitivity analysis justified?

29. Were the ranges over which the parameters were varied stated?

30. Were relevant alternatives compared? (That is, were appropriate comparisons made when conducting the incremental analysis?)

31. Was an incremental analysis reported?

32. Were major outcomes presented in a disaggregated as well as aggregated form?

33. Was the answer to the study question given?

34. Did conclusions follow from the data reported?

35. Were conclusions accompanied by the appropriate caveats?

36. Were generalisability issues addressed?

Adapted from Drummond MF, Jefferson TO (1996) Guidelines for authors and peer reviewers of economic submissions to the BMJ. The BMJ Economic Evaluation Working Party. British Medical Journal 313 (7052): 275-83. Cited in Centre for Reviews and Dissemination (2008) Systematic reviews. CRD's guidance for undertaking reviews in health care. York: Centre for Reviews and Dissemination

\section{Drummond (2005) classification scheme for economic evaluations}

\begin{tabular}{|c|c|c|c|c|}
\hline \multirow{6}{*}{$\begin{array}{l}\text { Are two or more } \\
\quad \text { alternatives compared? }\end{array}$} & \multirow[t]{4}{*}{ No } & No & & Yes \\
\hline & & Examine consequences only & $\begin{array}{l}\text { Examine } \\
\text { costs only }\end{array}$ & \\
\hline & & PARTIAL EVALUATION & & $\begin{array}{l}\text { PARTIAL } \\
\text { EVALUATION }\end{array}$ \\
\hline & & Outcome description (1A) & $\begin{array}{l}\text { Cost } \\
\text { description } \\
\text { (1B) }\end{array}$ & $\begin{array}{l}\text { Cost-outcome description } \\
\text { (2) }\end{array}$ \\
\hline & \multirow[t]{2}{*}{ Yes } & PARTIAL EVALUATION & & $\begin{array}{l}\text { FULL ECONOMIC } \\
\text { EVALUATION (4) }\end{array}$ \\
\hline & & $\begin{array}{l}\text { Efficacy effectiveness } \\
\text { evaluation (e.g. RCT) (3A) }\end{array}$ & $\begin{array}{l}\text { Cost analysis } \\
\text { (3B) }\end{array}$ & $\begin{array}{l}\text { Cost-effectiveness } \\
\text { analysis } \\
\text { Cost-utility analysis } \\
\text { Cost-benefit analysis }\end{array}$ \\
\hline
\end{tabular}




\section{Risk of bias assessments of randomised controlled trials-criteria for judging risk of bias assessments}

\section{Item}

1. Random sequence generation (selection bias)

2. Allocation concealment (selection bias)

3. Blinding of participants and providers (performance bias) Objective outcomes

\begin{abstract}
Judgement Description
Low risk The investigators describe a random component in the sequence generation process such as random number table; computer random number generator; coin tossing; shuffling cards or envelopes; throwing dice; drawing of lots; minimisation.
\end{abstract}

High risk The investigators describe a non-random component in the sequence generation process such as odd or even date of birth; date (or day) of admission; hospital or clinic record number; alternation; judgement of the clinician; results of a laboratory test or a series of tests; availability of the intervention.

Unclear Insufficient information about the sequence risk generation process to permit judgement of low or high risk.

Low risk Investigators enrolling participants could not foresee assignment because one of the following, or an equivalent method, was used to conceal allocation: central allocation (including telephone, web-based, and pharmacy-controlled randomisation); sequentially numbered drug containers of identical appearance; sequentially numbered, opaque, sealed envelopes.

High risk Investigators enrolling participants could possibly foresee assignments because one of the following method was used: open random allocation schedule (e.g. a list of random numbers); assignment envelopes without appropriate safeguards (e.g. if envelopes were unsealed or non-opaque or not sequentially numbered); alternation or rotation; date of birth; case record number; any other explicitly unconcealed procedure.

Unclear Insufficient information to permit judgement of risk low or high risk. This is usually the case if the method of concealment is not described or not described in sufficient detail to allow a definite judgement.

Low risk No blinding or incomplete blinding, but the review authors judge that the outcome is not likely to be influenced by lack of blinding; blinding of participants and key study personnel ensured, and unlikely that the blinding could have been broken.

High risk No blinding or incomplete blinding, and the outcome is likely to be influenced by lack of 
4. Blinding of participants and providers (performance bias) - subjective outcomes

5. Blinding of outcome assessor (detection bias) - objective outcomes
6.Blinding of outcome assessor (detection bias) - subjective outcomes blinding; blinding of key study participants and personnel attempted, but likely that the blinding could have been broken, and the outcome is likely to be influenced by lack of blinding.

Unclear Insufficient information to permit judgement of risk low or high risk; the study did not address this outcome.

Low risk Blinding of participants and providers and unlikely that the blinding could have been broken;

High risk No blinding or incomplete blinding, and the outcome is likely to be influenced by lack of blinding; blinding of key study participants and personnel attempted, but likely that the blinding could have been broken, and the outcome is likely to be influenced by lack of blinding.

Unclear Insufficient information to permit judgement of risk low or high risk; the study did not address this outcome.

Low risk No blinding of outcome assessment, but the review authors judge that the outcome measurement

is not likely to be influenced by lack of blinding; blinding of outcome assessment ensured, and unlikely that the blinding could have been broken.

High risk No blinding or incomplete blinding, and the outcome is likely to be influenced by lack of blinding; blinding of key study participants and personnel attempted, but likely that the blinding could have been broken, and the outcome is likely to be influenced by lack of blinding.

Unclear Insufficient information to permit judgement of risk low or high risk; the study did not address this outcome.

Low risk No blinding of outcome assessment, but the review authors judge that the outcome measurement is not likely to be influenced by lack of blinding; blinding of outcome assessment ensured, and unlikely that the blinding could have been broken.

High risk No blinding of outcome assessment, and the outcome measurement is likely to be influenced by lack of blinding; blinding of outcome assessment, but likely that the blinding could have been broken, and the outcome measurement is likely to be influenced by lack of blinding.

Unclear Insufficient information to permit judgement of risk low or high risk.

Low risk No missing outcome data; reasons for missing outcome data unlikely to be related to true 
7. Incomplete outcome data (attrition bias)for all outcomes except retention in treatment or drop out
8. Selective reporting (reporting bias) outcome (for survival data, censoring unlikely to be introducing bias); missing outcome data balanced in numbers across intervention groups, with similar reasons for missing data across groups; for dichotomous outcome data, the proportion of missing outcomes compared with observed event risk not enough to have a clinically relevant impact on the intervention effect estimate; for continuous outcome data, plausible effect size (difference in means or standardised difference in means) among missing outcomes not enough to have a clinically relevant impact on observed effect size; missing data have been imputed using appropriate methods; all randomised patients are reported/analysed in the group they were allocated to by randomisation irrespective of non-compliance and co-interventions (intention to treat)

High risk Reason for missing outcome data likely to be related to true outcome, with either imbalance in numbers or reasons for missing data across intervention groups;

for dichotomous outcome data, the proportion of missing outcomes compared with observed event risk enough to induce clinically relevant bias in intervention effect estimate; for continuous outcome data, plausible effect size (difference in means or standardised difference in means) among missing outcomes enough to induce clinically relevant bias in observed effect size; "As-treated" analysis done with substantial departure of the intervention received from that assigned at randomisation.

Unclear Insufficient information to permit judgement of risk low or high risk (e.g. number randomised not stated, no reasons for missing data provided; number of drop out not reported for each group).

Low risk The study protocol is available and all of the study's pre-specified (primary and secondary) outcomes that are of interest in the review have been reported in the pre-specified way; the study protocol is not available but it is clear that the published reports include all expected outcomes, including those that were pre-specified (convincing text of this nature may be uncommon).

High risk Not all of the study's pre-specified primary outcomes have been reported; one or more primary outcomes is reported using measurements, analysis methods or subsets of the data (e.g. sub scales) that were not pre-specified. 
Unclear risk

9. Other bias
One or more reported primary outcomes were not pre-specified (unless clear justification for their reporting is provided, such as an unexpected adverse effect); one or more outcomes of interest in the review are reported incompletely so that they cannot be entered in a meta-analysis; the study report fails to include results for a key outcome that would be expected to have been reported for such a study.

Low risk Evidence to suggest other problems identified with the study which might threaten the validity of the random allocation, attrition or data integrity and results of the trial.

High risk Evidence to suggest that the trial might be underpowered/problems with the random allocation process leading to potential self-selection bias/ issues of analysis not conducted using intent to treat analysis or evidence of missing data; concerns of attrition and measurement error including reliance on self-reported measures.

Unclear Insufficient information to permit judgement of risk low or high risk.

\section{Risk of bias of RCTs: summary of the assessments}

\section{Risk of bias domain summaries}

Random sequence Although all the included studies were randomised trials, nearly 50\% (10/22) provided an inadequate description and were rated as unclear by the reviewers (Brodie et al., 2009, Brown et al., 2013, Cornish et al., 1997, Coviello et al., 2010, Hanlon et al., 1975, Hanlon et al., 1977, Howells et al., 2002, Kinlock et al., 2005, Kurland et al., 1975, Parmar et al., 2017). Eleven studies were reported at low risk of bias (Bayanzadeh, 2004, Dolan et al., 2003, Dole et al., 1969, Kinlock et al., 2007, Konstenius et al., 2014, Lee et al., 2016, Lobmaier et al., 2010, Magura et al., 2009, McKenzie et al., 2012, Rich et al., 2015, Wright et al., 2011) and the remaining study was at high risk of bias (Cropsey et al., 2011).

Allocation concealment

Of the 22 included studies, five reported that the allocation process was concealed and were rated at low risk of bias (Dolan et al., 2003, Konstenius et al., 2014, Lee et al., 2016, Lobmaier et al., 2010, Wright et al., 2011). Five studies were rated at high risk of bias (Bayanzadeh, 2004, Cropsey et al., 2011, Magura et al., 2009, McKenzie et al., 2012, Rich et al., 2015). The remaining 12 studies were all rated as unclear (Brodie et al., 2009, Brown et al., 2013, Cornish et al., 1997, Dole et al., 1969, Hanlon et al., 1975, Hanlon et al., 1977, Howells et al., 2002, Kinlock et al., 2005, Kinlock et al., 2007, Konstenius et al., 2014, Kurland et al., 1975, Parmar et al., 2017) and the review authors were unable to decide whether allocation concealment had occurred within the studies.

Blinding

Blinding was assessed across four dimensions considering performance and detection bias across subjective and objective measures. Seven studies were rated as unclear risk of bias and provided no information on blinding across all four domains (Bayanzadeh, 2004, Brown et al., 2013, Cropsey et al., 2011, Dole et al., 1969, 
Kinlock et al., 2005, Kinlock et al., 2007, Magura et al., 2009). Eight studies were rated at high risk of bias for participant and personnel blinding (Cornish et al., 1997, Coviello et al., 2010, Dolan et al., 2003, Lee et al., 2016, Lobmaier et al., 2010, McKenzie et al., 2012, Rich et al., 2015, Wright et al., 2011). Seven studies reported use of a double-blind trial methodology and were rated low risk of bias (Brodie et al., 2009, Hanlon et al., 1975, Hanlon et al., 1977, Howells et al., 2002, Konstenius et al., 2014, Kurland et al., 1975, Parmar et al., 2017).

Incomplete outcome Three studies were noted at low risk of bias (Cornish et al., 1997, Dole et al., 1969, data Kinlock et al., 2007) and 18 studies were noted to be at high risk of bias (Bayanzadeh, 2004, Brodie et al., 2009, Brown et al., 2013, Coviello et al., 2010, Cropsey et al., 2011, Dolan et al., 2003, Hanlon et al., 1975, Hanlon et al., 1977, Howells et al., 2002, Kinlock et al., 2005, Konstenius et al., 2014, Lee et al., 2016, Lobmaier et al., 2010, Magura et al., 2009, McKenzie et al., 2012, Parmar et al., 2017, Rich et al., 2015, Wright et al., 2011). One study was rated as unclear risk of bias (Kurland et al., 1975).

Selective reporting Of the 20 studies, six studies were rated as unclear (Bayanzadeh, 2004, Cropsey et al., 2011, Dole et al., 1969, Hanlon et al., 1977, Kinlock et al., 2005, Kurland et al., 1975) and twelve studies were rated at low risk (Cornish et al., 1997, Coviello et al., 2010, Dolan et al., 2003, Howells et al., 2002, Kinlock et al., 2007, Konstenius et al., 2014, Lee et al., 2016, Lobmaier et al., 2010, Magura et al., 2009, McKenzie et al., 2012, Rich et al., 2015, Wright et al., 2011). Four studies were rated at high risk of bias (Brodie et al., 2009, Brown et al., 2013, Hanlon et al., 1975, Parmar et al., 2017).

\section{Risk of bias of RCTs: detailed assessments}

\section{Bayanzadeh 2004}

\section{Bias}

Random sequence generation (selection bias)
Allocation concealment (selection bias)

Blinding of participants and personnel Unclear risk (performance bias) subjective outcomes

Blinding of participants and personnel Unclear risk (performance bias) objective outcomes

Blinding of outcome assessment (detection bias) subjective outcomes

Blinding of outcome assessment (detection bias) objective outcomes
High risk

\section{Review \\ authors' \\ judgement}

Low risk

Unclear risk

Unclear risk

High risk

\section{Support for judgement}

Participants were categorised into one of four lists based on their previous history of drug abuse. The random allocation was then chosen, using even and odd row numbers from each list

The lists generated with stratification were probably not concealed

No information reported

No information on blinding reported

No information on blinding reported

No information on blinding reported 
Incomplete outcome data (attrition

bias) all outcomes

Selective reporting (reporting bias)
Unclear risk
After random allocation, 20 participants from the control group opted out of the research. At the end of the study attrition was high in both groups: for the intervention group $n=38$ out of the original 60 allocated and for the control group $n=31$ out of the original 60 allocated.

\section{Brodie 2009}

Bias

Random sequence generation (selection bias)

Allocation concealment (selection bias)

Blinding of participants and personnel Low risk (performance bias) subjective outcomes

Blinding of participants and personnel Low risk (performance bias) objective outcomes

Blinding of outcome assessment (detection bias) subjective outcomes

Blinding of outcome assessment (detection bias) objective outcomes

Incomplete outcome data (attrition bias) all outcomes

Selective reporting (reporting bias)

\section{Review authors' judgement}

Unclear risk

Unclear risk

Unclear risk

Unclear risk

High risk

High risk

\section{Support for judgement}

Method for random sequence generation not reported

Method for allocation of participants to treatment groups not reported

Staff, and personnel were both blind to treatment.

Staff and personnel were both blind to treatment

Blinding of outcome assessors not reported

Blinding of outcome assessors not reported

$38 \%$ in the vigabatrin group and $58.5 \%$ in the placebo group were lost to follow-up. Outcomes reported for $n$ randomised. Missing urine toxicology tests were considered positive.

Protocol identified for trial registration NCT00527683. Primary and secondary outcomes are clearly stated but some secondary mental health outcomes are not reported at follow-up.

\section{Brown 2013}

Bias

Random sequence generation (selection bias)

Allocation concealment (selection bias)

Blinding of participants and personnel Unclear risk (performance bias) subjective outcomes

Blinding of participants and personnel Unclear risk (performance bias) objective outcomes

\section{Review \\ authors' judgement}

Unclear risk Random allocation noted no further information.

Unclear risk No information provided

No information provided

No information provided 


\begin{tabular}{|c|c|c|}
\hline $\begin{array}{l}\text { Blinding of outcome assessment } \\
\text { (detection bias) subjective } \\
\text { outcomes }\end{array}$ & Unclear risk & No information provided \\
\hline $\begin{array}{l}\text { Blinding of outcome assessment } \\
\text { (detection bias) objective outcomes }\end{array}$ & Unclear risk & No information provided \\
\hline $\begin{array}{l}\text { Incomplete outcome data (attrition } \\
\text { bias) all outcomes }\end{array}$ & High risk & $\begin{array}{l}80 \% \text { completing the } 24 \text {-week assessment, } 33 \% \\
\text { completing week } 52 \text { and } 26 \% \text { at follow-up. }\end{array}$ \\
\hline Selective reporting (reporting bias) & High risk & $\begin{array}{l}\text { Protocol reported as being available. However, } \\
\text { on-going drug use (frequency and patterns of daily } \\
\text { drug use), health services utilisation and urine } \\
\text { tests are reported as being assessed, but no out- } \\
\text { come data are reported. }\end{array}$ \\
\hline
\end{tabular}

\begin{tabular}{|c|c|c|}
\hline \multicolumn{3}{|l|}{ Cornish 1997} \\
\hline Bias & $\begin{array}{l}\text { Review } \\
\text { authors' } \\
\text { judgement }\end{array}$ & Support for judgement \\
\hline $\begin{array}{l}\text { Random sequence generation (selection } \\
\text { bias) }\end{array}$ & Unclear risk & $\begin{array}{l}\text { Individuals were assigned at a ratio of } 2: 1 \text { to } \\
\text { naltrexone vs control }\end{array}$ \\
\hline Allocation concealment (selection bias) & Unclear risk & Not reported \\
\hline $\begin{array}{l}\text { Blinding of participants and personnel } \\
\text { (performance bias) subjective outcomes }\end{array}$ & High risk & $\begin{array}{l}\text { Study description suggests that participants } \\
\text { were not blind }\end{array}$ \\
\hline $\begin{array}{l}\text { Blinding of participants and personnel } \\
\text { (performance bias) objective outcomes }\end{array}$ & High risk & $\begin{array}{l}\text { Study description suggests that participants } \\
\text { were not blind }\end{array}$ \\
\hline $\begin{array}{l}\text { Blinding of outcome assessment (detection } \\
\text { bias) subjective outcomes }\end{array}$ & Unclear risk & $\begin{array}{l}\text { No information was reported on whether the } \\
\text { subjective measures were blinded }\end{array}$ \\
\hline $\begin{array}{l}\text { Blinding of outcome assessment (detection } \\
\text { bias) objective outcomes }\end{array}$ & Low risk & Outcome assessors were blind \\
\hline $\begin{array}{l}\text { Incomplete outcome data (attrition bias) all } \\
\text { outcomes }\end{array}$ & Low risk & ITT analyses completed. \\
\hline
\end{tabular}

Selective reporting (reporting bias)

Low risk

Study protocol referred to within the paper

\section{Coviello 2010}

Bias

Random sequence generation (selection bias)

Allocation concealment (selection bias)

Blinding of participants and personnel High risk (performance bias) subjective outcomes

Blinding of participants and personnel High risk (performance bias) objective outcomes

Blinding of outcome assessment (detection bias) subjective outcomes

\section{Review Support for judgement \\ authors' judgement}

Unclear risk Randomisation method unclear. Note that randomisation was balanced by using six variables

Unclear risk No information provided

The treatment as usual group were not blinded

The treatment as usual group were not blinded

Unclear risk No information was reported on whether the subjective measures were blinded 


\begin{tabular}{|c|c|c|}
\hline $\begin{array}{l}\text { Blinding of outcome assessment } \\
\text { (detection bias) objective outcomes }\end{array}$ & Unclear risk & $\begin{array}{l}\text { No information was reported on whether the } \\
\text { objective measures were blinded }\end{array}$ \\
\hline $\begin{array}{l}\text { Incomplete outcome data (attrition } \\
\text { bias) all outcomes }\end{array}$ & High risk & $\begin{array}{l}\text { A large amount of attrition was noted in the first } \\
\text { week, and only one-third of participants remained } \\
\text { at 6-month follow-up }\end{array}$ \\
\hline Selective reporting (reporting bias) & Low risk & Study protocol referred to in the paper \\
\hline
\end{tabular}

\section{Cropsey 2011}

\section{Bias}

Random sequence generation (selection bias)

\author{
Allocation concealment (selection \\ bias) \\ Blinding of participants and personnel Unclear risk \\ (performance bias) subjective \\ outcomes \\ High risk
}

Blinding of participants and personnel Unclear risk (performance bias) objective outcomes
Blinding of outcome assessment (detection bias) subjective outcomes

Blinding of outcome assessment (detection bias) objective outcomes

Incomplete outcome data (attrition bias) all outcomes

Selective reporting (reporting bias)

High risk

\section{Review \\ authors' \\ judgement}

(n)
double-blind trial of participants and providers on all outcomes. Some concerns about contamination issues with the placebo group but difficult to assess to what extent the blinding might have been affected.

This trial began as an open label trial then became a double-blind trial of participants and providers on all outcomes. Some concerns about contamination issues with the placebo group but difficult to assess to what extent the blinding might have been affected.

Unclear risk No evidence to provide information about whether the assessors who conducted the outcome assessments were blind

Unclear risk No evidence to provide information about whether the assessors who conducted the outcome assessments were blind

Unclear risk A total of 8 individuals (22\%) were not included in the final analysis after randomisation. It is unclear whether an ITT analyses was conducted.

\section{High risk No protocol identified.}

\section{Dolan 2003}

\section{Bias}

Random sequence generation (selection bias)

\section{Review Support for judgement \\ authors' judgement}

Low risk Group allocation was based on block randomisation. A sequential list of case numbers was matched to group allocations in blocks of ten by randomly drawing five cards labelled "control" and five cards labelled "treatment" from an envelope. This procedure was repeated for each block of ten 


\begin{tabular}{|c|c|c|}
\hline $\begin{array}{l}\text { Allocation concealment (selection } \\
\text { bias) }\end{array}$ & Low risk & $\begin{array}{l}\text { Allocation held by researcher not involved in } \\
\text { recruiting or interviewing participants. Trial } \\
\text { nurses had no access to lists }\end{array}$ \\
\hline $\begin{array}{l}\text { Blinding of participants and personnel } \\
\text { (performance bias) subjective } \\
\text { outcomes }\end{array}$ & High risk & $\begin{array}{l}\text { Treatment and comparator (methadone or wait list) } \\
\text { would not permit blinding }\end{array}$ \\
\hline $\begin{array}{l}\text { Blinding of participants and personnel } \\
\text { (performance bias) objective } \\
\text { outcomes }\end{array}$ & High risk & $\begin{array}{l}\text { Treatment and comparator (methadone or wait list) } \\
\text { would not permit blinding }\end{array}$ \\
\hline $\begin{array}{l}\text { Blinding of outcome assessment } \\
\text { (detection bias) subjective } \\
\text { outcomes }\end{array}$ & Unclear risk & No information was provided \\
\hline $\begin{array}{l}\text { Blinding of outcome assessment } \\
\text { (detection bias) objective outcomes }\end{array}$ & Unclear risk & No information was provided \\
\hline $\begin{array}{l}\text { Incomplete outcome data (attrition } \\
\text { bias) all outcomes }\end{array}$ & High risk & $\begin{array}{l}\text { Attrition }>30 \% \text { in both groups and ITT not } \\
\text { undertaken. At follow-up, } 129(68 \%) \text { treated and } \\
124(65 \%) \text { control subjects who had been in } \\
\text { continuous custody were re-interviewed. } 29 \\
\text { treated and } 33 \text { control subjects had been released } \\
\text { from prison and were excluded. No data on other } \\
\text { participants not accounted for at follow-up }\end{array}$ \\
\hline Selective reporting (reporting bias) & Low risk & Protocol information matched all outcome measures \\
\hline
\end{tabular}

sequential case numbers. The list of case numbers and group allocation was held by a researcher not involved in recruiting or interviewing inmates. The trial nurses responsible for assessing, recruiting and interviewing inmates had no access to these lists.

\section{Dole 1969}

Bias

Random sequence generation (selection bias) Low risk

Allocation concealment (selection bias)

Blinding of participants and personnel (performance bias) subjective outcomes

Blinding of participants and personnel (performance bias) objective outcomes

Blinding of outcome assessment (detection bias) subjective outcomes

Blinding of outcome assessment (detection bias) objective outcomes

Incomplete outcome data (attrition bias) all outcomes

Selective reporting (reporting bias)

\section{Review authors' Support for judgement judgement}

Allocation by lottery, no further details of the study method provided

Unclear risk Not reported

Unclear risk No information provided

Unclear risk No information provided

Unclear risk No information provided

Unclear risk No information provided

Low risk No attrition reported on any of the key outcome measures

Unclear risk Protocol for the trial not identified.

\section{Hanlon 1975}




\begin{tabular}{|c|c|c|}
\hline & $\begin{array}{l}\text { Review } \\
\text { authors' } \\
\text { judgement }\end{array}$ & \\
\hline $\begin{array}{l}\text { Random sequence generation } \\
\text { (selection bias) }\end{array}$ & Unclear risk & Reported as randomly assigned no details provided \\
\hline $\begin{array}{l}\text { Allocation concealment (selection } \\
\text { bias) }\end{array}$ & Unclear risk & Not reported \\
\hline $\begin{array}{l}\text { Blinding of participants and personnel } \\
\text { (performance bias) subjective } \\
\text { outcomes }\end{array}$ & Low risk & Reported as double-blind study \\
\hline $\begin{array}{l}\text { Blinding of participants and personnel } \\
\text { (performance bias) objective } \\
\text { outcomes }\end{array}$ & Low risk & Reported as double-blind study \\
\hline $\begin{array}{l}\text { Blinding of outcome assessment } \\
\text { (detection bias) subjective } \\
\text { outcomes }\end{array}$ & Unclear risk & Not reported in the study \\
\hline $\begin{array}{l}\text { Blinding of outcome assessment } \\
\text { (detection bias) objective outcomes }\end{array}$ & Unclear risk & Not reported in the study \\
\hline $\begin{array}{l}\text { Incomplete outcome data (attrition } \\
\text { bias) all outcomes }\end{array}$ & High risk & $\begin{array}{l}\text { Attrition across the two groups was high. In the } \\
\text { intervention group } 21(60 \%) \text { people did not } \\
\text { complete the intervention and in the comparison } \\
\text { intervention } 17(48.6 \%) \text { did not complete the } \\
\text { intervention. No intent to treat analysis is } \\
\text { provided. }\end{array}$ \\
\hline Selective reporting (reporting bias) & High risk & $\begin{array}{l}\text { Primary and secondary outcomes are not reported } \\
\text { separately. No trial protocol is reported. The } \\
\text { results were divided into those who completed and } \\
\text { those who did not complete the intervention. }\end{array}$ \\
\hline
\end{tabular}

\section{Hanlon 1977}

Bias

Random sequence generation (selection bias)

Allocation concealment (selection bias)

Blinding of participants and personnel Low risk (performance bias) subjective outcomes

Blinding of participants and personnel Low risk (performance bias) objective outcomes

Blinding of outcome assessment (detection bias) subjective outcomes

Blinding of outcome assessment (detection bias) objective outcomes

Incomplete outcome data (attrition bias) all outcomes

\section{Review \\ authors' \\ judgement}

Unclear risk Not reported

Unclear risk Not reported

Reported as a double-blind trial

Reported as a double-blind trial

Unclear risk Not reported

Unclear risk Not reported

High risk
Of the original 154 parolees referred and assigned to one of the two treatment arms -57 did not receive study medication and were excluded. This reduced the resulting sample size on which the 
analyses were performed to 55 in the intervention group and 42 in the placebo group.

Selective reporting (reporting bias) Unclear risk Not reported — no evidence of a trial protocol

\section{Howells 2002}

Bias

Random sequence generation (selection bias)

Allocation concealment (selection bias)

Blinding of participants and personnel Low risk (performance bias) subjective outcomes

Blinding of participants and personnel Low risk (performance bias) objective outcomes

Blinding of outcome assessment Low risk (detection bias) subjective outcomes

Blinding of outcome assessment Low risk (detection bias) objective outcomes

Incomplete outcome data (attrition bias) all outcomes

Selective reporting (reporting bias) Low risk

\section{Support for judgement}

authors'

The authors report "The pharmacist who made up the medication used a simple randomisation procedure to allocate each participant to one arm of the trial" but no further description is reported.

Unclear risk

The authors report "The independent pharmacy team at the prison oversaw the randomisation and blinding procedure...", but no statement that allocation was concealed

"...both the patient and health centre clinicians were blind to the assigned treatment group"

"...both the patient and health centre clinicians were blind to the assigned treatment group"

"...blinding was maintained during treatment of the patients and during data entry and analysis"

"...blinding was maintained during treatment of the patients and during data entry and analysis"

Twenty-one participants (27.63\%) (13/32 lofexidine, $8 / 36$ methadone) were withdrawn from the trial prematurely. ITT not used, data analysed per-protocol

The authors indicate that there was a protocol for the study ("Patient safety elements in the protocol were as follows:"') and primary and secondary outcomes are clearly defined. Outcome data for the primary and secondary outcomes are reported

\section{Kinlock 2005}

Bias

Random sequence generation (selection bias)

Allocation concealment (selection bias)

Blinding of participants and personnel (performance bias) subjective outcomes

Blinding of participants and personnel Unclear risk (performance bias) objective outcomes

Unclear risk

\section{Review authors' judgement}

Unclear risk No information reported other than stated 'random'

No information reported

No information reported

No information reported 


$\begin{array}{ccc}\begin{array}{c}\text { Blinding of outcome assessment } \\ \text { (detection bias) subjective outcomes }\end{array} & \text { Unclear risk } & \text { No information reported } \\ \begin{array}{c}\text { Blinding of outcome assessment } \\ \text { (detection bias) objective outcomes }\end{array} & \text { Unclear risk } & \text { No information reported } \\ \begin{array}{c}\text { Incomplete outcome data (attrition } \\ \text { bias) all outcomes }\end{array} & \text { High risk } & \text { Follow-up interviews were conducted on } 66, \text { or } \\ & & 80 \% \text { of the } 83 \text { participants who were eligible; } 22 \\ & & \text { of 26 experimental participants (85\%), 31 of 38 } \\ & \text { control participants (82\%), and 13 of } 19 \text { who } \\ & & \text { declined participation in the experimental } \\ & \text { condition (68\%). }\end{array}$

Selective reporting (reporting bias) Unclear risk No protocol recorded.

\section{Kinlock 2007}

Bias

Random sequence generation (selection bias)

Allocation concealment (selection bias)

Blinding of participants and personnel Unclear risk (performance bias) subjective outcomes

Blinding of participants and personnel Unclear risk (performance bias) objective outcomes

Blinding of outcome assessment (detection bias) subjective outcomes

Blinding of outcome assessment (detection bias) objective outcomes

Incomplete outcome data (attrition Low risk bias) all outcomes

Selective reporting (reporting bias) Low risk

\section{Review \\ authors' \\ judgement}

Low risk Block randomisation

Unclear risk No information reported

No information reported

No information reported

Unclear risk No information reported

Unclear risk No information reported

One month post-release follow-up assessments were conducted on 200 , or $96.2 \%$ of the 208 participants due for this assessment; 64 of $70(91.4 \%)$ in the counselling only condition, 66 of $68(97.1 \%)$ in the counselling + transfer condition, and 70 of $70(100.0 \%)$ in the counselling + methadone condition

Study protocol referenced and supported outcome measures.

\section{Konstenius 2014}

Bias

Random sequence generation (selection bias)

Allocation concealment (selection bias)

\section{Review \\ authors' \\ judgement}

Low risk

Low risk

\section{Support for judgement}

The randomisation list was generated by an independent pharmacist using the computer-based programme design by Trombult Programming between March 2007 and February 2011. 
Blinding of participants and personnel Low risk (performance bias) subjective outcomes

Blinding of participants and personnel Low risk (performance bias) objective outcomes

Blinding of outcome assessment (detection bias) subjective outcomes

Blinding of outcome assessment (detection bias) objective outcomes

Incomplete outcome data (attrition High risk bias) all outcomes

Selective reporting (reporting bias) Low risk
The randomisation code was retained by the Karolinska Pharmacy and disclosed after the end of the trial. No interim analysis was performed.

Although efforts were made to maintain blinding, $48 \%$ of the participants receiving MPH and $48 \%$ of the placebo group identified their medication correctly during the titration phase or after reaching the maximum dose.

Although efforts were made to maintain blinding, $48 \%$ of the participants receiving $\mathrm{MPH}$ and $48 \%$ of the placebo group identified their medication correctly during the titration phase or after reaching the maximum dose.

Unclear risk Not reported if assessor was blind

Unclear risk Not reported if assessor was blind

High risk $>10 \%$ in both groups did not complete the trial, more in the placebo group than the intervention group.

The trial was registered in the International Standard Randomised Controlled Trial Number Register (ISRCTN) at http:// www.controlled-trials. com/ISRCTN77940178.

\begin{tabular}{|c|c|c|}
\hline \multicolumn{3}{|l|}{ Kurland 1975} \\
\hline Bias & $\begin{array}{l}\text { Review } \\
\text { authors' } \\
\text { judgement }\end{array}$ & Support for judgement \\
\hline Random sequence generation (selection bias) & Unclear risk & Not reported \\
\hline Allocation concealment (selection bias) & Unclear risk & Not reported \\
\hline $\begin{array}{l}\text { Blinding of participants and personnel } \\
\text { (performance bias) subjective outcomes }\end{array}$ & Low risk & Reported as double-blind trial \\
\hline $\begin{array}{l}\text { Blinding of participants and personnel } \\
\text { (performance bias) objective outcomes }\end{array}$ & Low risk & Reported as double-blind trial \\
\hline $\begin{array}{l}\text { Blinding of outcome assessment (detection } \\
\text { bias) subjective outcomes }\end{array}$ & Unclear risk & Not reported \\
\hline $\begin{array}{l}\text { Blinding of outcome assessment (detection } \\
\text { bias) objective outcomes }\end{array}$ & Unclear risk & Not reported \\
\hline $\begin{array}{l}\text { Incomplete outcome data (attrition bias) all } \\
\text { outcomes }\end{array}$ & Unclear risk & $\begin{array}{l}\text { Reporting is given by group but no } \\
\text { reasons for withdrawal are provided }\end{array}$ \\
\hline Selective reporting (reporting bias) & Unclear risk & No evidence of a protocol provided \\
\hline
\end{tabular}

Lee 2016

Review
authors'
judgement

Random sequence generation

Low risk (selection bias) 
Allocation concealment (selection Low risk bias)

Blinding of participants and personnel High risk (performance bias) subjective outcomes

Blinding of participants and personnel High risk (performance bias) objective outcomes

Blinding of outcome assessment High risk (detection bias) subjective outcomes

Blinding of outcome assessment High risk (detection bias) objective outcomes

Incomplete outcome data (attrition High risk bias) all outcomes

Selective reporting (reporting bias) Low risk
An independent, centralised, automated telephone system made the treatment assignments after eligibility of the participants was confirmed

Sealed envelopes were sequenced and shuffled. Participants and researchers could not foresee assignment.

This is an open trial no blinding was conducted

This is an open trial no blinding was conducted

This is an open trial no blinding was conducted

This is an open trial no blinding was conducted

$13 \%$ were lost to follow-up in the naltrexone group and $8 \%$ in usual care, and some outcomes were only available for completers.

Trial protocol NCT 00781898 is registered and outcomes are divided clearly into primary and secondary outcomes

\section{Lobmaier 2010}

\section{Bias}

Random sequence generation (selection bias)

Allocation concealment (selection bias)

Blinding of participants and personnel High risk (performance bias) subjective outcomes

Blinding of participants and personnel High risk (performance bias) objective outcomes

Blinding of outcome assessment (detection bias) subjective outcomes

Blinding of outcome assessment (detection bias) objective outcomes

Incomplete outcome data (attrition bias) all outcomes

\section{Review \\ authors' \\ judgement}

Low risk

Low risk

Unclear risk

Unclear risk

High risk

\section{Support for judgement}

Treatment allocation sequence performed at an independent centre using a permuted block protocol

Sequentially numbered, sealed, opaque envelopes

"the treatment conditions were not blind and may have increased risk of performance bias"

"the treatment conditions were not blind and may have increased risk of performance bias"

No information provided

No information provided

In the naltrexone implant arm of the trial, 7 of 23 inmates did not initiate treatment: all 7 reported a preference for methadone or a non-study treatment. In the methadone treatment arm, 10 of 21 inmates did not initiate treatment and dropped out before release: $60 \%$ of the methadone group drop-outs reported that they intended to start, but were not granted the possibility to continue with 
methadone maintenance upon release by community treatment providers.

Selective reporting (reporting bias) Low risk The study was funded by the Research Council of Norway and registered publicly at http://clinicaltrials.gov, identifier NCT00204243. All primary and secondary outcomes reported

\section{Magura 2009}

Bias

Random sequence generation (selection bias)

Allocation concealment (selection bias)

High risk

Blinding of participants and personnel Unclear risk (performance bias) subjective outcomes

Blinding of participants and personnel Unclear risk (performance bias) objective outcomes

Blinding of outcome assessment (detection bias) subjective outcomes

Blinding of outcome assessment (detection bias) objective outcomes

Incomplete outcome data (attrition High risk bias) all outcomes

Selective reporting (reporting bias) Low risk

Low risk

\section{Review Support for judgement}

Unclear risk No information provided

Unclear risk No information provided

Some attrition occurred before medication was received by buprenorphine-assigned participants. Interview follow-ups were attempted for 60 buprenorphine subjects, of whom 43 (72\%) were interviewed at follow-up, and for 56 methadone subjects, of whom 38 (68\%) were interviewed.

The study was approved by the Institutional Review Boards of National Development and Research Institutes (NDRI) and DOHMH. The ClinicalTrials.gov identifier is NCT00367302. Protocol listed all primary and secondary outcomes

\section{McKenzie 2012}

\section{Bias}

Random sequence generation (selection bias)

Allocation concealment (selection bias)

\section{Review \\ authors' \\ judgement}

Low risk

High risk

High risk

\section{Support for judgement}

Computer-generated random permutation. Urn randomisation to stratify on the basis of sex and race.

Open-label trial no mention of blinding

Open-label trial no mention of blinding 
Blinding of participants and personnel (performance bias) subjective outcomes

Blinding of participants and personnel High risk (performance bias) objective outcomes

Blinding of outcome assessment (detection bias) subjective outcomes

Blinding of outcome assessmen (detection bias) objective outcomes

Incomplete outcome data (attrition High risk bias) all outcomes

Selective reporting (reporting bias) Low risk
Open-label trial no mention of blinding

Unclear risk The assessment of outcomes was not described

Unclear risk The assessment of outcomes was not described

High risk There was substantial cross over from arms 1 and 3 to 2 . The authors suggested that the treatment completer analysis is more reliable than the ITT analysis. Data for the completers only are presented.

Protocol and trial is registered see NCT00142935. All outcomes are reported as expected.

\section{Parmar 2016}

Bias

Random sequence generation (selection bias)

Allocation concealment (selection bias)

Blinding of participants and personnel Low risk (performance bias) subjective outcomes

Blinding of participants and personnel Low risk (performance bias) objective outcomes

\section{Blinding of outcome assessment (detection bias) subjective outcomes}

Blinding of outcome assessment (detection bias) objective outcomes

Incomplete outcome data (attrition bias) all outcomes

Selective reporting (reporting bias)

\section{Review \\ authors' judgement}

Unclear risk Randomisation by MRC trials unit. No further information provided

Unclear risk Allocation concealment not reported

The trial was double-blind prior to release so that, whilst the participant was still in custody and pre-release, neither the participant, prison-based N-ALIVE staff nor prison staff knew the allocation. Participants learned their allocation when they opened the pack at the time of their release.

The trial was double-blind prior to release so that, whilst the participant was still in custody and pre-release, neither the participant, prison-based N-ALIVE staff nor prison staff knew the allocation. Participants learned their allocation when they opened the pack at the time of their release.

High risk

The self-reported questionnaire identified the participant's randomised assignment and the time interval between the preceding N-ALIVE release date and completion date.

Unclear risk Not applicable - no objective measures reported in the trial.

High risk Approximately 20\% attrition in each group - due to those allocated did not get their allocation as intended.

High risk Study protocol is reported but not all of the study's pre-specified primary outcomes have been reported due to the trial stopping early. Protocol (ISRCTN34044390) 


\section{Rich 2015}

Bias

Random sequence generation (selection bias)

Allocation concealment (selection bias)

Blinding of participants and personnel High risk (performance bias) subjective outcomes

Blinding of participants and personnel High risk (performance bias) objective outcomes

Blinding of outcome assessment (detection bias) subjective outcomes

Blinding of outcome assessment (detection bias) objective outcomes

Incomplete outcome data (attrition High risk bias) all outcomes

Selective reporting (reporting bias) High risk

\section{Support for judgement}

authors'

judgement

Low risk Computer generated random permutation. Urn randomisation to stratify on the basis of sex and race. Independent of enrolment and consent process. Random assignment was obtained from a staff member who had no direct contact with participants

No blinding conducted using an open-label trial design

No blinding conducted using an open-label trial design

No blinding conducted using an open-label trial design

Unclear risk Blinding of outcome measures not clearly reported

Unclear risk Blinding of outcome measures not clearly reported

In the methadone continuation group data were available for $114 / 142(80 \%)$. In the tapered withdrawal group data were available for 109/141 $(77 \%)$. There is some attrition.

Study protocol is reported and all of specified outcomes are measured Protocol (NCT01874964)

\section{Wright 2011}

\section{Bias}

Random sequence generation (selection bias)

Allocation concealment (selection bias)

Blinding of participants and personnel High risk (performance bias) subjective outcomes

Blinding of participants and personnel High risk (performance bias) objective outcomes

Low risk

Low risk

\section{Review Support for judgement \\ authors' \\ judgement} function

Sealed, opaque, consecutively numbered envelopes concealing the name of the allocated intervention were prepared by a researcher who had no contact with participants.

Open label "The prescribing doctor randomised by opening the next envelope and prescribing the intervention named inside. Both prisoner and doctor were blind to the intervention until this point".

Open label "The prescribing doctor randomised by opening the next envelope and prescribing the intervention named inside. Both prisoner and doctor were blind to the intervention until this point". 


\begin{tabular}{|c|c|c|}
\hline $\begin{array}{l}\text { Blinding of outcome assessment } \\
\text { (detection bias) subjective } \\
\text { outcomes }\end{array}$ & Unclear risk & $\begin{array}{l}\text { No statement regarding blinding of individual who } \\
\text { undertook the biochemical urine tests. }\end{array}$ \\
\hline $\begin{array}{l}\text { Blinding of outcome assessment } \\
\text { (detection bias) objective outcomes }\end{array}$ & Unclear risk & $\begin{array}{l}\text { No statement regarding blinding of individual who } \\
\text { recorded self-reported or clinical notes. }\end{array}$ \\
\hline $\begin{array}{l}\text { Incomplete outcome data (attrition } \\
\text { bias) all outcomes }\end{array}$ & High risk & $\begin{array}{l}\text { High levels of attrition. } 50 \% \text { buprenorphine and } 45 \% \\
\text { methadone did not provide urine sample at day } 8 \text {. } \\
65 \% \text { and } 62 \% \text { at } 1 \text { month, } 80 \% \text { and } 85 \% \text { at } \\
3 \text { months and } 86 \% \text { and } 91 \% \text { at } 6 \text { months. }\end{array}$ \\
\hline Selective reporting (reporting bias) & Low risk & $\begin{array}{l}\text { Published study protocol identified. All primary and } \\
\text { secondary outcomes reported. }\end{array}$ \\
\hline
\end{tabular}

Open Access This article is licensed under a Creative Commons Attribution 4.0 International License, which permits use, sharing, adaptation, distribution and reproduction in any medium or format, as long as you give appropriate credit to the original author(s) and the source, provide a link to the Creative Commons licence, and indicate if changes were made. The images or other third party material in this article are included in the article's Creative Commons licence, unless indicated otherwise in a credit line to the material. If material is not included in the article's Creative Commons licence and your intended use is not permitted by statutory regulation or exceeds the permitted use, you will need to obtain permission directly from the copyright holder. To view a copy of this licence, visit http://creativecommons.org/licenses/by/4.0/.

\section{References}

Amato, L., Davoli, M., Perucci, C. A., Ferri, M., Faggiano, F., \& Mattick, R. P. (2005). An overview of systematic reviews of the effectiveness of opiate maintenance therapies: available evidence to inform clinical practice and research. Journal of Substance Abuse Treatment, 28(4), 321-329.

Bayanzadeh SA. (2004). Final report of research project: a study of the effectiveness of psychopharmacological and psychological interventions in reducing harmful/high risk behaviours among substance user prisoners. Iran University of Medical Education and Health and Treatment Services, Tehran Psychology Institute. Centre for Psychological Health Research Polarity of Science, Education and Research.

Binswanger, I., Stern, M., Deyo, R., Heagerty, P., Cheadle, A., Elmore, J., et al. (2007). Release from prison a high risk of death for former inmates. New England Journal of Medicine, 356(2), 157-165.

Bird, S. M., \& Hutchinson, S. J. (2003). Male drugs-related deaths in the fortnight after release from prison: Scotland, 1996-99. Addiction, 98(2), 185-190.

Brodie, J. D., Case, B. G., Figueroa, E., Dewey, S. L., Robinson, J. A., Wanderling, J. A., et al. (2009). Randomized, double-blind, placebo-controlled trial of vigabatrin for the treatment of cocaine dependence in Mexican parolees. American Journal of Psychiatry, 166(11), 1269-1277.

Brooke, D., Taylor, C., Gunn, J., \& Maden, A. (1996). Point prevalence of mental disorder in unconvicted male prisoners in England and Wales. BMJ, 313(7071), 1524-1527.

Brown, R., Gassman, M., Hetzel, S., \& Berger, L. (2013). Community-based treatment for opioid dependent offenders: a pilot study. American Journal on Addictions, 22(5), 500-502.

Catania, H. (2003). Prison health needed in prisons. Harm reduction news: newsletter of the International Harm Reduction Development Program of the Open Society Institute. Open Society Institute, 4(11), 13.

Chanhatasilpa, C., MacKenzie, D. L., \& Hickman, L. J. (2000). The effectiveness of community-based programs for chemically dependent offenders: a review and assessment of the research. Journal of Substance Abuse Treatment, 19(4), 383-393.

Clarivate (2020). EndNote X9.3.3 for Windows \& Mac [program]. (Philadelphia: Clarivate Analytics).

Cornish, J. W., Metzger, D., Woody, G. E., Wilson, D., McLellan, A. T., Vandergrift, B., et al. (1997). Naltrexone pharmacotherapy for opioid dependent federal probationers. Journal of Substance Abuse Treatment, 14(6), 529-534. 
Coviello, D. M., Cornish, J. W., Lynch, K. G., Alterman, A. I., \& O'Brien, C. P. (2010). A randomized trial of oral naltrexone for treating opioid-dependent offenders. American Journal on Addictions, 19(5), 422-432.

Cropsey, K. L., Lane, P. S., Hale, G. J., Jackson, D. O., Clark, C. B., Ingersoll, K. S., et al. (2011). Results of a pilot randomized controlled trial of buprenorphine for opioid dependent women in the criminal justice system. Drug and Alcohol Dependence, 119(3), 172-178.

Deeks, J. J., Higgins, J. P. T. \& Altman, D. G. (2017). Chapter 9: analysing data and undertaking metaanalyses. In J. P. T. Higgins et al. (Eds.), Cochrane handbook for systematic reviews of interventions (version 5.2.0) [updated June 2017]. Cochrane Collaboration. https://training.cochrane.org/handbook/ archive/v5.2. Accessed 29 Jan 2021.

Dolan, K. A., Shearer, J., MacDonald, M., Mattick, R. P., Hall, W., \& Wodak, A. D. (2003). A randomised controlled trial of methadone maintenance treatment versus wait list control in an Australian prison system. Drug and Alcohol Dependence, 72(1), 59-65.

Dole, V. P., Robinson, J. W., Orraca, J., Towns, E., Searcy, P., \& Caine, E. (1969). Methadone treatment of randomly selected criminal addicts. New England Journal of Medicine, 280(25), 1372-1375.

Drummond, M., Sculpher, M., Torrance, G., O'Brien, B., \& Stoddart, G. (2005). Methods for the economic evaluation of health care programmes (3rd ed.). Oxford: Oxford University Press.

Egg, R., Pearson, F. S., Cleland, C. M., \& Lipton, D. S. (2000). Evaluations of correctional treatment programs in Germany: a review and meta-analysis. Substance Use and Misuse, 35(12-14), 1967-2009.

Faggiano, F., Vigna-Taglianti, F., Versino, E., \& Lemma, P. (2003). Methadone maintenance at different dosages for opioid dependence. Cochrane Database of Systematic Reviews, 3(1), CD002208.

Fazel, S., Bains, P., \& Doll, H. (2006). Substance abuse and dependence in prisoners: a systematic review. Addiction, 101(2), 181-191.

Fazel, S., Yoon, I., \& Hayes, A. (2015). Substance use disorders in prisoners: an updated systematic review and meta-regression analysis in recently incarcerated men and women. Addiction, 112(10), 1725-1739.

Fiscella, K., Moore, A., Engerman, J., \& Meldrum, S. (2004). Jail management of arrestees/inmates enrolled in community methadone maintenance programs. Journal of Urban Health, 81(4), 645-654.

Friedmann, P., Wilson, D., Hoskinson, R., Poshkus, M., \& Clarke, J. (2018). Initiation of extended release naltrexone (XR-NTX) for opioid use disorder prior to release from prison. Journal of Substance Abuse Treatment, 85, 45-48.

Garcia, C., Correa, G., Viver, A., Hernandez, B., Kinlock, T., Gordon, M., et al. (2007). Buprenorphinenaloxone treatment for pre-release opioid-dependent inmates in Puerto Rico. Journal of Addiction Medicine, 1, 126-132.

Gibson, A. E., \& Degenhardt, L. J. (2007). Mortality related to pharmacotherapies for opioid dependence: a comparative analysis of coronial records. Drug and Alcohol Review, 26(4), 405-410.

Gordon, M. S., Kinlock, T. W., Schwartz, R. P., O'Grady, K. E., Fitzgerald, T. T., \& Vocci, F. J. (2017). A randomized clinical trial of buprenorphine for prisoners: findings at 12-months post-release. Drug and Alcohol Dependence, 172, 34-42.

Gunn, J., Maden, A., \& Swinton, M. (1991). Treatment needs of prisoners with psychiatric disorders. BMJ, 303(6798), 338-341.

Hanlon, T. E., McCabe, O. L., Savage, C., \& Kurland, A. A. (1975). A controlled comparison of cyclazocine and naloxone treatment of the paroled narcotic addict. International Pharmacopsychiatry, 10(4), 240250 .

Hanlon, T. E., McCabe, O. L., Savage, C., \& Kurland, A. A. (1977). Narcotic antagonist treatment of addict parolees - the failure of an effective approach. Comprehensive Psychiatry, 18(3), 211-219.

Hedrich, D., Alves, P., Farrell, M., Stover, H., Moller, L., \& Mayet, S. (2012). The effectiveness of opioid maintenance treatment in prison settings: a systematic review. Addiction, 107(3), 501-517.

Higgins, J. P. T. \& Green, S. (2011). Cochrane handbook for systematic reviews of interventions (version 5.1.0) [updated March 2011]. The Cochrane Collaboration. https://handbook-5-1.cochrane.org/. Accessed 29 Jan 2021.

Howells, C., Allen, S., Gupta, J., Stillwell, G., Marsden, J., \& Farrell, M. (2002). Prison based detoxification for opioid dependence: a randomised double blind controlled trial of lofexidine and methadone. Drug and Alcohol Dependence, 67(2), 169-176.

Kinlock, T., Battjes, R., Schwartz, R., \& Team, M. P. (2005). A novel opioid maintenance program for prisoners: report of postrelease outcomes. American Journal of Drug and Alcohol Abuse, 31(3), 433-454.

Kinlock, T. W., Gordon, M. S., Schwartz, R. P., O'Grady, K., Fitzgerald, T. T., \& Wilson, M. (2007). A randomized clinical trial of methadone maintenance for prisoners: results at 1-month post-release. Drug and Alcohol Dependence, 91(2-3), 220-227.

Kinner, S. A., Forsyth, S., \& Williams, G. (2013). Systematic review of record linkage studies of mortality in ex-prisoners: why (good) methods matter. Addiction, 108(1), 38-49. 
Klientkartlaggningen, K. (2015). Ett regeringsuppdrag (1st ed.). Norrköping.

Konstenius, M., Jayaram-Lindstrom, N., Guterstam, J., Beck, O., Philips, B., \& Franck, J. (2014). Methylphenidate for attention deficit hyperactivity disorder and drug relapse in criminal offenders with substance dependence: a 24-week randomized placebo-controlled trial. Addiction, 109(3), 440-449.

Kurland, A. A., McCabe, L., \& Hanlon, T. E. (1975). Contingent naloxone (n-allylnoroxymorphone) treatment of the paroled narcotic addict. International Pharmacopsychiatry, 10(3), 157-168.

Lee, J. D., Friedmann, P. D., Kinlock, T. W., Nunes, E. V., Gordon, M. S., \& O’Brien, C. P. (2015). Extended-release naltrexone for opioid relapse prevention among opioid-dependent, criminal justiceinvolved adults. Drug and Alcohol Dependence, 156, e125-e125.

Lee, J. D., Friedmann, P. D., Kinlock, T. W., Nunes, E. V., Boney, T. Y., Hoskinson, R. A., et al. (2016). Extended-release naltrexone to prevent opioid relapse in criminal justice offenders. New England Journal of Medicine, 374(13), 1232-1242.

Lo, C. C., \& Stephens, R. C. (2000). Drugs and prisoners: treatment needs on entering prison. American Journal of Drug and Alcohol Abuse, 26(2), 229-245.

Lobmaier, P., Kornor, H., Kunoe, N., \& Bjorndal, A. (2008). Sustained-release naltrexone for opioid dependence. Cochrane Database of Systematic Reviews, 2, CD006140.

Lobmaier, P. P., Kunoe, N., Gossop, M., Katevoll, T., \& Waal, H. (2010). Naltrexone implants compared to methadone: outcomes six months after prison release. European Addiction Research, 16(3), 139-145.

Magura, S., Kang, S. Y., \& Shapiro, J. L. (1995). Measuring cocaine use by hair analysis among criminally involved youth. Journal of Drug Issues, 25, 683-701.

Magura, S., Lee, J. D., Hershberger, J., Joseph, H., Marsch, L., Shropshire, C., et al. (2009). Buprenorphine and methadone maintenance in jail and post-release: a randomized clinical trial. Drug and Alcohol Dependence, 99(1-3), 222-230.

Marsch, L. A. (1998). The efficacy of methadone maintenance interventions in reducing illicit opiate use, HIV risk behaviours and criminality: a meta-analysis. Addiction, 93(4), 515-532.

Maruschak, L. M., \& Minton, T. D. (2020). Correctional populations in the United States, 2017-2018 [online]. Washington DC: Bureau of Justice Statistics, Department of Justice Available at: https://www. bjs.gov/content/pub/pdf/cpus1718.pdf.

Mason, D., Birmingham, L., \& Grubin, D. (1997). Substance use in remand prisoners: a consecutive case study. BMJ, 315, 18-21.

Mattick, R. P., Breen, C., Kimber, J., \& Davoli, M. (2009). Methadone maintenance therapy versus no opioid replacement therapy for opioid dependence. Cochrane Database of Systematic Reviews, 3, CD002209.

McKenzie, M., Zaller, N., Dickman, S., Green, T., Parihk, A., Friedmann, M., et al. (2012). A randomized trial of methadone initiation prior to release from incarceration. Substance Abuse, 33(1), 19-20.

Merrall, E. L., Kariminia, A., Binswanger, I. A., Hobbs, M. S., Farrell, M., Marsden, J., et al. (2010). Metaanalysis of drug-related deaths soon after release from prison. Addiction, 105(9), 1545-1554.

Michel, L., \& Maguet, O. (2005). Guidelines for substitution treatments in prison populations. Encephale, 31(1 Pt 1), 92-97.

Minozzi, S., Amato, L., Vecchi, S., Davoli, M., Kirchmayer, U., \& Verster, A. (2011). Oral naltrexone maintenance treatment for opioid dependence. Cochrane Database of Systematic Reviews, 4, CD001333.

Mitchell, O., Wilson, D., \& MacKenzie, D. (2006). The effectiveness of incarceration-based drug treatment on criminal behaviour. Campbell Systematic Reviews, 2(1), 1-56.

Mitchell, O., MacKenzie, L., \& Wilson, D. (2012a). The effectiveness of incarcerated based drug treatment on criminal behaviour: a systematic review. Campbell Systematic Reviews, 8(1), 1-76.

Mitchell, O., Wilson, D., Eggers, A., \& Mackenzie, L. D. (2012b). Drug courts effects on criminal offending for juveniles and adults: a systematic review. Campbell Systematic Reviews, 8(1), 1-87.

Moller, L., Gathere, A., Juergens, R., Stover, H., \& Nikogosian, H. (2007). Health in prisons: a WHO guide to the essentials in prison health. Copenhagen: World Health Organization Regional Office for Europe.

Murphy, S. M., Polsky, D., Lee, J. D., Friedmann, P. D., Kinlock, T. W., Nunes, E. V., et al. (2017). Costeffectiveness of extended release naltrexone to prevent relapse among criminal justice-involved individuals with a history of opioid use disorder. Addiction, 112(8), 1440-1450.

National Institute for Health and Care Excellence (NICE). (2007a). NICE technology appraisal guidance 114: methadone and buprenorphine for the management of opioid dependence [online]. London: NICE Available at: www.nice.org.uk/TA114.

National Institute for Health and Care Excellence (NICE). (2007b). NICE technology appraisal guidance 115: naltrexone for the management of opioid dependence [online]. London: NICE Available at: www.nice. org.uk/TA115. 
National Institute for Health and Care Excellence (NICE). (2019). NICE technology appraisal guidance opioid dependence: buprenorphine prolonged release injection [online]. London: NICE Available at: https://www.nice.org.uk/advice/es19/chapter/Key-messages.

National Institute on Drug Abuse (NIDA). (2020). Criminal justice drug facts [online]. Available at: https:// www.drugabuse.gov/sites/default/files/drugfacts-criminal-justice.pdf. Accessed 4 Nov 2020.

Parmar, M. K. B., Strang, J., Choo, L., Meade, A. M., \& Bird, S. M. (2017). Randomized controlled pilot trial of naloxone-on-release to prevent post-prison opioid overdose deaths. Addiction, 112(3), 502-515.

Perry, A., Coulton, S., Glanville, J., Godfrey, C., Lunn, J., McDougall, C., et al. (2006). Interventions for drugusing offenders in the courts, secure establishments and the community. Cochrane Database of Systematic Reviews, 3, CD005193.

Perry, A. E., Neilson, M., Martyn-St James, M., Glanville, J. M., Woodhouse, R., Godfrey, C., et al. (2015). Pharmacological interventions for drug-using offenders. Cochrane Database of Systematic Reviews, 2(6), CD010862.

Ramstedt, M., Sundin, E., Landberg, J., \& Raninen, J. (2013). ANTD-bruket och dess negativa konsekvenser I befolkninge (1st ed.). Stockholm: Stockholm förebygger Alkohol och Drogproblem.

Reynaud-Maurupt, C., Caer, Y., Escaffre, N., Gagneau, M., Galinier, A., Marzo, J. N., et al. (2005). High-dose buprenorphine substitution during incarceration. Presse Médicale, 34(7), 487-490.

Rich, J., McKenzie, M., Larney, S., Wong, J., Tran, L., \& Clarke, J. (2015). Methadone continuation versus forced withdrawal on incarceration in a combined US prison and jail: a randomised, open-label trial. Lancet, 386(9991), 350-359.

Stallwitz, A., \& Stover, H. (2007). The impact of substitution treatment in prisons - a literature review. International Journal of Drug Policy, 18(6), 464-474.

Strang, J., Kelleher, M., Best, D., Mayet, S., \& Manning, V. (2006). Preventing heroin overdose deaths with emergency naloxone -new legislation broadens scope of potential providers and contexts. BMJ, 333, 614 615 .

The Nordic Cochrane Centre (The Cochrane Collaboration). (2014). Review manager (RevMan). The Cochrane Collaboration: Copenhagen.

Veritas Health Innovation. (2013). Covidence systematic review software. Melbourne: Veritas Health Innovation. Systematic Review Software.

Warren, E., Viney, R., Shearer, J., Shanahan, M., Wodak, A., \& Dolan, K. (2006). Value for money in drug treatment: economic evaluation of prison methadone. Drug and Alcohol Dependence, 84(2), 160-166.

Weisburd, D. (2010). Justifying the use of non-experimental methods and disqualifying the use of randomized controlled trials: Challenging folklore in evaluation research in crime and justice. Journal of Experimental Criminology, 6, 209-227.

Wright, N. M. J., Sheard, L., Adams, C. E., Rushforth, B. J., Harrison, W., Bound, N., et al. (2011). Comparison of methadone and buprenorphine for opiate detoxification (Leeds trial): A randomised controlled trial. British Journal of General Practice, 61(593), e772-e780.

Publisher's note Springer Nature remains neutral with regard to jurisdictional claims in published maps and institutional affiliations. 
J. M. Glanville Julie is a qualified librarian who has worked in systematic reviews for more than 25 years and is an independent consultant focusing on information retrieval for systematic reviews. From 2008 to June 2020, she was Associate Director of York Health Economics Consortium (YHEC) and coordinated its information and review services.

A. E. Perry is a Senior Lecturer and Chartered Forensic Psychologist in the Mental Health and Addictions team in the Department of Health Sciences at the University of York. Amanda's research interest includes the application of psychological evidence to support people involved in the criminal justice system.

M. Martyn-St James is a systematic reviewer with a proven track record of applying many types of systematic review and meta-analyses methods in various research areas.

C. Hewitt is a Professor of Trials and Statistics and Deputy Director of York Trials Unit. Her research has focused on developing, refining and applying statistical methods in the conduct of randomised controlled trials and systematic reviews in a variety of clinical areas.

S. Swarmi is a health economist with experience varying from academia and industry. She holds a Master's and a Bachelor's degree in Economics. She is currently a health economic consultant at Evidera, London. Prior to Evidera, she was working as a Research Fellow at the Department of Health Sciences, University of York.

K. Wright worked as an Information Service Manager since 1997. Her role involved supporting research staff in their work and, in particular, carrying out literature searching for systematic reviews and health technology assessments.

L. Burns is a secondary English teacher with a Master's in Public Health from the University of York.

C. Pearson is a Psychological Wellbeing Practitioner in Research at Tees Esk and Wear Valley NHS Trust. She is completing her final year of the Counselling Psychology Doctorate at Teesside University. Her work focuses on consent and patient/ service user rights.

A. Aboaja is a consultant forensic psychiatrist and a New Care Models clinical director for adult secure care in the North East and North Cumbria. Her research interests include sleep, global prison mental health and PPIE (patient and public involvement and engagement)

P. Thakkar is a consultant forensic psychiatrist at Rampton High Secure Hospital. His interests are offender mental health, neurobiology of violence and medical leadership.

K. M. S. Kumar works as a Senior Clinical Director and Consultant Forensic Psychiatrist in TEWV NHS Trust. My additional qualifications are MBBS from India and a PG Diploma in Forensic Mental Health. I am specifically interested in embedding neurosciences within the Bio-psycho-social model of psychiatry and medicine alike. Towards a "brainful and mindful" person centred, psychiatry.

M. Bunney is a Project Administrator at York Health Economics Consortium (YHEC). Matthew's role at YHEC involves providing project assistance and administrative support in systematic reviews. 


\section{Affiliations}

J. M. Glanville ${ }^{1}$ - A. E. Perry ${ }^{2} \cdot$ M. Martyn-St James ${ }^{3} \cdot$ C. Hewitt ${ }^{2} \cdot$ S. Swami ${ }^{6} \cdot$ K. Wright $^{5} \cdot$ L. Burns ${ }^{2}$ - C. Pearson ${ }^{2}$ - A. Aboaja ${ }^{4}$ - P. Thakkar ${ }^{4}$ - K. M. S. Kumar ${ }^{4}$ - M. Bunney ${ }^{1}$

1 York Health Economics Consortium, York, UK

2 Department of Health Sciences, University of York, Heslington, York YO10 5DD, UK

3 School of Health and Related Research (ScHARR), University of Sheffield, Sheffield, UK

4 Tees, Esk and Wear Valleys NHS Foundation Trust, Middlesbrough, UK

5 Centre for Reviews and Dissemination, York, UK

6 Evidera, London, UK 